\title{
Weyl's Problem for the Spectral Distribution of Laplacians on P.C.F. Self-Similar Fractals
}

\author{
Jun Kigami and Michel L. Lapidus ${ }^{2 \star}$ \\ 1 Department of Mathematics, College of General Education, Osaka University, Toyonaka 560, \\ Japan. e-mail: f61531a@center.osaka-u-ac.jp \\ 2 Department of Mathematics, Sproul Hall, The University of California, Riverside, CA 92521-0135, \\ USA. e-mail: Lapidus@ucrmath.ucr.edu
}

Received June 22, 1992; in revised form January 25, 1993

\begin{abstract}
We establish an analogue of Weyl's classical theorem for the asymptotics of eigenvalues of Laplacians on a finitely ramified (i.e., p.c.f.) self-similar fractal $K$, such as, for example, the Sierpinski gasket. We consider both Dirichlet and Neumann boundary conditions, as well as Laplacians associated with Bernoulli-type ("multifractal") measures on $K$. From a physical point of view, we study the density of states for diffusions or for wave propagation in fractal media. More precisely, let $\varrho(x)$ be the number of eigenvalues less than $x$. Then we show that $\varrho(x)$ is of the order of $x^{d_{S} / 2}$ as $x \rightarrow+\infty$, where the "spectral exponent" $d_{S}$ is computed in terms of the geometric as well as analytic structures of $K$. Further, we give an effective condition that guarantees the existence of the limit of $x^{-d_{S} / 2} \varrho(x)$ as $x \rightarrow+\infty$; this condition is, in some sense, "generic". In addition, we define in terms of the above "spectral exponents" and calculate explicitly the "spectral dimension" of $K$.
\end{abstract}

\section{Introduction}

In this paper, we will study the asymptotic behavior of the spectrum of the Laplacians on some self-similar sets. This problem occurs naturally in the study of physical phenomena, such as waves and diffusions, on fractal objects.

At first, we recall Weyl's classical result. Let $\Omega$ be a bounded domain in $\mathbb{R}^{n}$, with boundary $\partial \Omega$. We consider the following eigenvalue problem:

$$
\text { (DE) }\left\{\begin{array}{l}
\Delta u=-k u \text { on } \Omega, \\
\left.u\right|_{\partial \Omega}=0,
\end{array}\right.
$$

where $\Delta=\sum_{\imath=1}^{n} \partial^{2} / \partial x_{\imath}^{2}$ is the Laplacian on $\mathbb{R}^{n}$. It is well known that the eigenvalues - i.e., the scalars $k$ such that (DE) has a non-trivial solution $u$-are non-negative and

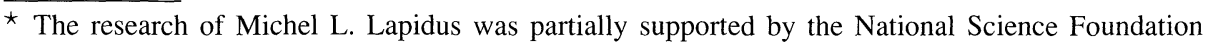
under Grant DMS-8904389 (transferred to the University of California as grant DMS-9196085) and Grant DMS-9207098, as well as by Yale University
} 
have finite multiplicity. Also they have only one accumulation point, namely $+\infty$. And hence we can define the eigenvalue counting function:

$$
\varrho_{0}(x)=\#\{k \mid k \text { is an eigenvalue no larger than } x\} .
$$

Of course, we are taking the multiplicity into account in the above definition. Then Weyl's famous asymptotic formula for the Dirichlet Laplacian is

Theorem 0.1. As $x \rightarrow+\infty$,

$$
\varrho_{0}(x)=(2 \pi)^{-n} \mathscr{S}_{n}|\Omega|_{n} x^{n / 2}+o\left(x^{n / 2}\right),
$$

where $|\Omega|_{n}$ denotes the $n$-dimensional Lebesgue measure (or "volume") of $\Omega$ and $\mathscr{B}_{n}$ is the volume of the unit ball in $\mathbb{R}^{n}$.

Remark. Weyl proved the above result under certain regularity conditions on the domains in [W1,2]. Now it has been shown for arbitrary bounded domains in $\mathbb{R}^{n}$. See Métivier [Me]. (See also Lapidus [La1-3] and the references therein and at the end of this introduction, for associated sharp error estimates (and related results) expressed in terms of the "fractal" (Minkowski) dimension of the boundary of $\Omega$.)

Our purpose in this paper is to obtain an analogue of the above result in the case of Laplacians on fractal sets.

There have been many early works about diffusions on fractals in physics. They were dealing, for example, with diffusions on percolation clusters and fractally structured media, currents on fractal electrical networks, as well as transport in porous media. For works in the physics literature on this subject, see in particular, Dhar [D], Alexander-Orbach [AO], Rammal-Toulouse [RT], and the references therein; see also the survey paper by Liu [Liu], as well as Havlin-Bunde [HB] and Chap. I, esp. pp. 4045 in Schroeder [Sc].

In mathematics, Kusuoka [Ku1], Goldstein [G] and Barlow-Perkins [BP], have defined and studied the "Brownian motion on the Sierpinski gasket." From their probabilistic point of view, "Brownian motion" is defined as a renormalized limit of random walks on the pre-gaskets (see Fig. 1), and the "Laplacian" is the infinitesimal generator of "Brownian motion."

Later, Kigami [Ki1] has defined the "Laplacian" on the Sierpinski gasket (S.G. for short) as a kind of renormalized limit of finite-difference operators on the pre-gaskets. We shall now briefly recall this analytical approach. Let the pre-gaskets $\left\{G_{m}\right\}_{m=1}^{\infty}$ be the sequence of finite graphs defined in Fig. 1a. The Sierpinski gasket is the closure of $\bigcup_{m \geq 0} V_{m}$, where $V_{m}$ is the set of vertices of $G_{m}$ (see Fig. $1 \mathrm{~b}$ and Example 2 in Sect. 3). The discrete Laplacian on $G_{m}$, denoted by $\Delta_{m}$, is a finite-difference operator defined as follows. For $f: V_{m} \rightarrow \mathbb{R}$ and $p \in V_{m}$,

$$
\left(\Delta_{m} f\right)_{p}=5^{m} \sum_{q \in V_{m, p}}(f(q)-f(p)),
$$

where $V_{m, p}$ is the set of vertices connected to $p$ by an edge of $G_{m}$. Then the Laplacian on the S.G. is defined as a limit of $\Delta_{m}$ by

$$
\Delta f=\lim _{m \rightarrow \infty} \Delta_{m} f .
$$

Of course, the former probabilistic approach and this definition have treated the same objects from different viewpoints. 

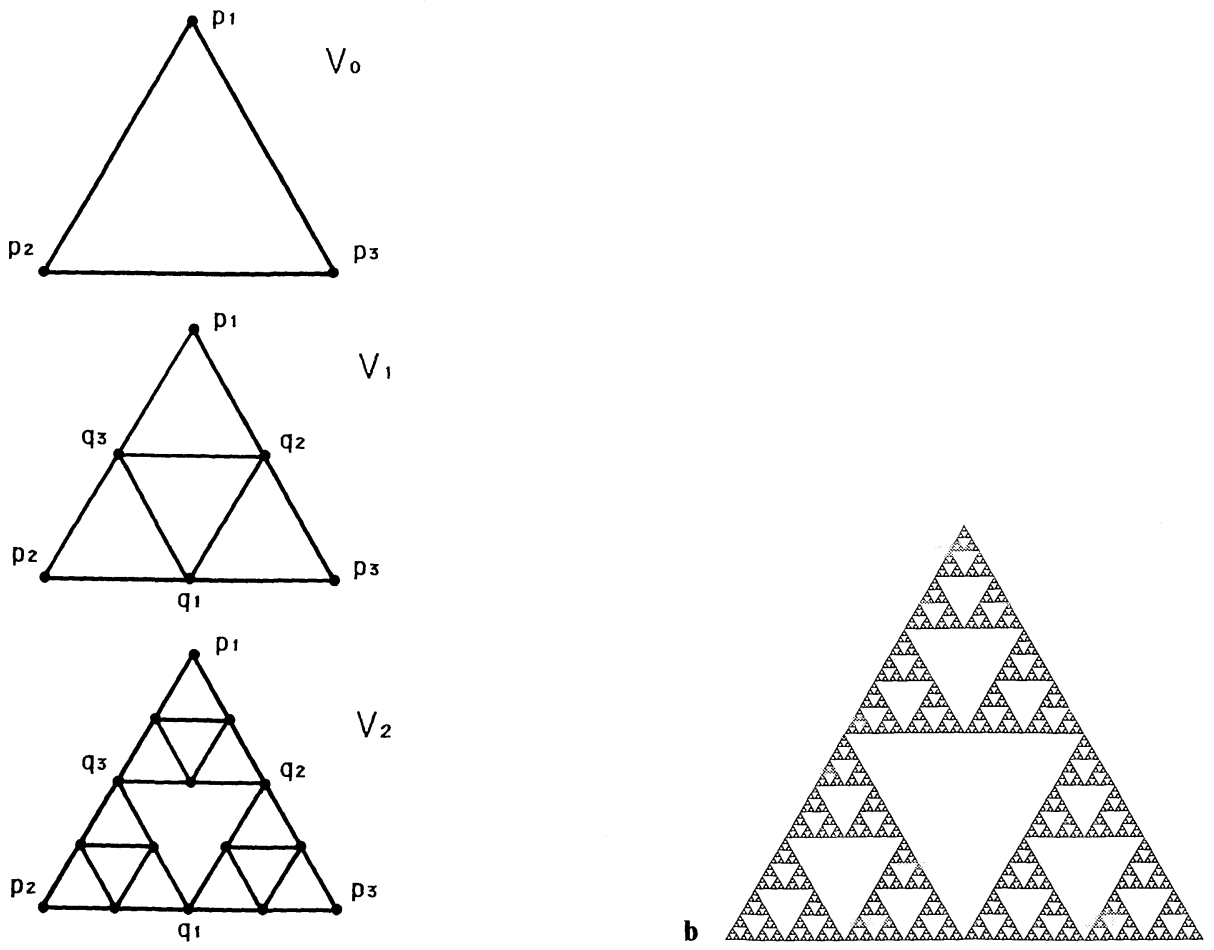

Fig. 1a. The Sierpinski pre-gaskets. $q_{1}=F_{3}\left(p_{2}\right)=F_{2}\left(p_{3}\right) ; q_{2}=F_{1}\left(p_{3}\right)=F_{3}\left(p_{1}\right) ; q_{3}=F_{2}\left(p_{1}\right)=$ $F_{1}\left(p_{2}\right)$. b. The Sierpinski gasket

Afterwards, these approaches have been extended to some classes of "finitely ramified" fractals: nested fractals by Lindstrøm [Li] (see Example 5 in Sect. 3) and postcritically finite (p.c.f. for short) self-similar sets by Kigami [Ki2]. As a result of the latter work, the Laplacian on the S.G. defined above should be called "the standard Laplacian on the S.G.," because it is now know that there are many other "Laplacians" on the Sierpinski gasket.

Now, what happens to the asymptotic behavior of the eigenvalue counting function on fractals? A natural analogue of Theorem 0.1 in that case could be stated as follows:

$$
\varrho_{0}(x)=C_{d} \mathscr{H}_{d}(\Omega) x^{\frac{d}{2}}+o\left(x^{\frac{d}{2}}\right) \quad \text { as } \quad x \rightarrow \infty,
$$

where $\Omega$ is a fractal set, $d=d_{H}$ is the Hausdorff dimension of $\Omega, \mathscr{H}_{d}$ is the $d$ dimensional Hausdorff measure and $C_{d}$ is a constant independent of $\Omega$. (For notational simplicity, we write " $x \rightarrow \infty$ " instead of " $x \rightarrow+\infty$ " throughout this paper.)

Indeed, the physicist Berry made such a conjecture in [Bel,2]. Unfortunately, this is wrong, as will be explained below.

The eigenvalues and eigenfunctions of the standard Laplacians on the S.G. have been determined exactly by Shima [Sh] and Fukushima-Shima [FS] by means of the eigenvalue decimation method introduced by Rammal-Toulouse [RT] and further studied by Rammal [Ra]. According to their result,

$$
0<\operatorname{liminv}_{x \rightarrow \infty} \varrho_{0}(x) x^{-\frac{d_{S}}{2}}<\limsup _{x \rightarrow \infty} \varrho_{0}(x) x^{-\frac{d_{S}}{2}}<\infty,
$$


where $d_{S}=\frac{\log 9}{\log 5}$ is called the "spectral dimension" of the S.G. In particular, there exist positive constants $c_{1}, c_{2}$ such that $c_{1} x^{d_{S} / 2} \leq \varrho_{0}(x) \leq c_{2} x^{d_{S} / 2}$, for all $x$ large enough.

From the probabilistic point of view, this exponent $d_{S}$ was previously obtained by Barlow and Perkins [BP] in the case of the S.G.

Before these mathematical works, physicists (Dhar [D], Alexander-Orbach [AO], Rammal-Toulouse [RT], Hattori-Hattori-Watanabe [HHW]) had obtained this value of the "spectral dimension" (also called "fracton dimension") from different points of view.

Comparing (0.2) with (0.1), the following two facts are remarkable and suggest several questions:

(1) $d_{S}=\log 9 / \log 5$ is not equal to the Hausdorff dimension of the S.G., $d_{H}=$ $\log 3 / \log 2$. How can we calculate the asymptotic order of the eigenvalue counting function of Laplacians on fractals?

(2) The limit as $x \rightarrow \infty$ of $\varrho_{0}(x) x^{-d_{S} / 2}$ does not exist. In general, does this kind of limit exist for Laplacians on fractals?

In this paper, we will give an answer to these questions for Laplacians on p.c.f. self-similar sets defined in [Ki2] and which will be introduced in Sect. 1.

The Laplacian on a p.c.f. self-similar set $K$ depends on the choice of a "harmonic structure" (Dirichlet form) and a probability measure $\mu$ on $K$. In this paper, we assume that $\mu$ is a Bernoulli (i.e., self-similar) measure.

Let $\varrho_{0}(x)$ be the eigenvalue counting function of the Laplacian on $K$, with Dirichlet boundary conditions. Our main result, Theorem 2.4, enables us in particular to determine the asymptotic order of $\varrho_{0}(x)$ as $x \rightarrow \infty$. More precisely, we show that there exist positive constants $c_{1}$ and $c_{2}$ such that

$$
c_{1} x^{\frac{d_{S}}{2}} \leq \varrho_{0}(x) \leq c_{2} x^{\frac{d_{S}}{2}}
$$

for all $x$ sufficiently large.

Here $d_{S}$, called the "spectral exponent of the Laplacian," is the unique positive number such that

$$
\sum_{i=1}^{N} \gamma_{i}^{d_{S}}=1
$$

where the positive numbers $\gamma_{2}$ are not the contraction ratios of $K$ but are determined by the harmonic structure and the probability measure $\mu$.

As an example of application, we deduce from (0.4) that $d_{S}<2$ for (regular) "finitely ramified fractals." of

Moreover, we give an effective condition that guarantees the existence of the limit

$$
\varrho_{0}(x) x^{-\frac{d_{S}}{2}}, \quad \text { as } \quad x \rightarrow \infty .
$$

Roughly speaking, our results show in particular that this limit exists (and is non-zero) for "almost all" Laplacians on a given p.c.f. self-similar set $K$.

We also obtain analogous results for Laplacians with Neumann boundary conditions (see Corollary 2.5).

The rest of this paper is organized as follows. In Sect. 1, we provide some of the necessary facts about p.c.f. self-similar sets. In Sect. 2, we state and prove our 
main results (Theorem 2.4 and Corollary 2.5) concerning the spectral distribution of Laplacians on $K$. In Sect. 3, we illustrate our theorems by discussing several examples. In Sects. 4-6, we then prove some key lemmas needed to establish our main results. [In particular, in Sects. 4 and 5, we study the variational approach to the Dirichlet and Neumann problems on $K$, as well as the consequences of (a refinement of) the method of "Dirichlet-Neumann bracketing" in this context. Further, in Sect. 6, we deduce from the self-similarity of $K$ a basic scaling property of the Dirichlet form (or "energy functional").] Finally, in an appendix, we define by means of the above "spectral exponents" the "spectral dimension" of $K$ and calculate it explicitly in terms of the geometric and analytic structures of $K$.

We close this introduction by mentioning several related results and motivations for the present work.

Barlow-Bass [BB1,2] have defined and studied a "Brownian motion" on the Sierpinski carpet, which is an "infinitely ramified" fractal and hence not p.c.f. The associated spectral dimension is shown to exist in $[\mathrm{BB} 3,4]$ and computed numerically in $[\mathrm{BBS}]$. (See also the review paper [Ba].)

From the point of view of the spectral distribution, there is an analogy - pointed out by Lapidus in [La4] - between the present situation and that of Laplacians on bounded open sets $\Omega \subset \mathbb{R}^{n}$ with "fractal" boundary $\partial \Omega$. In the latter case, under mild assumptions, the following sharp remainder estimate holds (see [La1]):

$$
\varrho_{0}(x)=\varphi(x)+O\left(x^{\frac{d_{M}}{2}}\right) \text { as } \quad x \rightarrow \infty
$$

where $\varphi(x)=(2 \pi)^{-n} \mathscr{S}_{n}|\Omega|_{n} x^{n / 2}$ is the leading term in Weyl's formula $(0.1)$ and $D \in(n-1, n)$ is the Minkowski dimension (also called Bouligand or "box" dimension) - rather than the Hausdorff dimension - of the boundary $\partial \Omega$. (This estimate is also established for more general elliptic operators as well as for Neumann boundary conditions in [La1, Theorems $2.1-3$ and 4.1, pp. 479-483 and 511]. A preTauberian form of $(0.5)$ (for the trace of the heat semigroup) was previously obtained for the Dirichlet Laplacian by Brossard-Carmona [BC]. See also [LF] and [La2].) Further, in certain specific cases, the behavior of the asymptotic second term of the eigenvalue counting function has been investigated by Lapidus-Pomerance in [LP13]. In particular, the limit as $x \rightarrow \infty$ of $\left(\varrho_{0}(x)-\varphi(x)\right) x^{d_{M} / 2}$ sometimes exists and sometimes does not. (See esp. [LP2, Examples 4.3 and 4.5, Sect. 4.3], the main example of [LP3], as well as Sects. 3-5 of the survey paper [La3]; see also [La4].) Partly motivated by these results and work of Lalley [L], a specific conjecture has been made by Lapidus in [La4] regarding the dichotomy for the existence of this limit for Laplacians on open sets with (suitable) self-similar fractal boundary. (See [La4, Conjecture 3, pp. 163-164, Sect. 4.4.1]. This conjecture was first made by the second author during a plenary address to the Regional Meeting of the American Mathematical Society held in Tampa, Florida, in March 1991 [La5].). The main result that we shall establish in the present paper for Laplacians on p.c.f. self-similar sets is very analogous to this conjecture. Further, it corrects, specifies and proves in this situation the counterpart of this conjecture (also made at the aforementioned meeting and stated in [La4, Conjecture 5, Remark 5.11(b), (c), pp. 189-190, Sect. 5.2]) for Laplacians on (suitable) self-similar fractals.

Finally, we note that in the terminology of [La1-5], our work deals with the vibrations of "drums with fractal membrane" rather than of "drums with fractal boundary," studied in particular in [BrCa, LF, La1-5, LP1-3, LM1-2]. 


\section{P.C.F. Self-Similar Sets}

In this section, we will briefly introduce post critically finite (p.c.f. for short) selfsimilar sets and Laplacians on them. The concept of p.c.f. self-similar sets is a mathematical justification of the notion of "finitely ramified" fractals. According to Hutchinson $[\mathrm{Hu}]$, a self-similar set is defined as a compact subset $K$ of a Euclidean space $\mathbb{R}^{n}$ such that

$$
K=\bigcup_{i=1}^{N} f_{i}(K),
$$

where $f_{1}, f_{2}, \ldots, f_{N}$ are contraction mappings from $\mathbb{R}^{n}$ to itself and $N$ is an integer $\geq 2$. In this terminology, finitely ramified fractals are roughly thought of as the selfsimilar sets $K$ such that $\bigcup_{\imath \neq j}\left(f_{i}(K) \cap f_{j}(K)\right)$ is a finite set. For instance, the Sierpinski gasket, a well-known example of self-similar set, is finitely ramified. In fact, for the Sierpinski gasket $K, \bigcup_{i \neq j}\left(f_{\imath}(K) \cap f_{j}(K)\right)$ consists of three points.

On the other hand, it is known that a self-similar set can be considered as a quotient space of one-sided shift spaces $\Sigma=\{1,2, \ldots, N\}^{\mathbb{N}}$ by a quotient map $\pi: \Sigma \rightarrow K$ defined by

$$
\{\pi(\omega)\}=\bigcap_{n \geq 1} f_{\omega_{1}} \circ f_{\omega_{2}} \circ \ldots \circ f_{\omega_{n}}(K)
$$

where $\omega=\omega_{1} \omega_{2} \omega_{3} \ldots$ In this manner, we can view a self-similar set as a purely topological object without the a priori assumption that it is embedded in some Euclidean space or that the mappings $f_{i}$ are contractions. This way of defining selfsimilar sets is natural for studying analysis on fractals because, as we will see later, Laplacians, Green's functions and Dirichlet forms depend only on the topological structure but not on the specific choice of metric.

We now define the notion of self-similar structure.

Definition 1.1. Let $K$ be a compact metrizable topological space and $\Sigma$ be the onesided shift space defined by $\Sigma=S^{\mathbb{N}}$, where $S=\{1,2, \ldots, N\}^{\mathbb{N}}$. Also, let $F_{2}$, for $i=1,2, \ldots, N$, be a continuous injection from $K$ to itself. Then, $\left(K, S,\left\{F_{i}\right\}_{\imath=1}^{N}\right)$ is called a self-similar structure if there exists a continuous surjection $\pi: \Sigma \rightarrow K$ such that $F_{\imath} \circ \pi=\pi \circ i$ for every $i=1,2, \ldots, N$, where $i: \Sigma \rightarrow \Sigma$ is defined by $i\left(\omega_{1} \omega_{2} \omega_{3} \ldots\right)=i \omega_{1} \omega_{2} \omega_{3} \ldots$

Remark 1. It is shown in [Ki2] that if (K,S, $\left.\left\{F_{i}\right\}_{\imath=1}^{N}\right)$ is a self-similar structure, then for all $\omega=\omega_{1} \omega_{2} \omega_{3} \ldots, \bigcap_{n>1} F_{\omega_{1}} \circ F_{\omega_{2}} \circ \ldots \circ F_{\omega_{2}}(K)$ consists of a single point and that $\pi$ is uniquely determined by $\{\pi(\omega)\}=\bigcap_{n \geq 1} F_{\omega_{1}} \circ F_{\omega_{2}} \circ \ldots \circ F_{\omega_{n}}(K)$.

Remark 2. There is a more general definition of self-similar set as a quotient space of $\Sigma$ by an equivalence relation $\sim$ such that $\omega \sim \omega^{\prime}$ implies $i \omega \sim i \omega^{\prime}$ for all $i=1, \ldots, N$. In this case, $\pi$ is the natural projection map from $\Sigma$ to $\Sigma / \sim$. In this setting, however, $\Sigma / \sim$ is not always metrizable. See Kusuoka [Ku2] and Kameyama [Ka].

Notation. $W_{m}=\{1,2, \ldots, N\}^{m}$ is the collection of words with length $m$. For $w=w_{1} w_{2} \ldots w_{m} \in W_{m}$, we define $F_{w}: K \rightarrow K$ by $F_{w}=F_{w_{1}} \circ F_{w_{2}} \circ \ldots \circ F_{w_{m}}$ and $K_{w}=F_{w}(K)$.

Next, we give the definition of a post critically finite (p.c.f.) self-similar structure. 
Definition 1.2. For a self-similar structure $\left(K, S,\left\{F_{\imath}\right\}_{i=1}^{N}\right)$, we define the critical set $\mathscr{C}$ and the post critical set $\mathscr{P}$ by $\mathscr{C}=\pi^{-1}\left(\bigcup_{\imath \neq j} F_{\imath}(K) \cap F_{j}(K)\right)$ and $\mathscr{P}=\bigcup_{n \geq 1} \sigma^{n}(\mathscr{C})$, where $\sigma: \Sigma \rightarrow \Sigma$ is the shift map given by $\sigma\left(\omega_{1} \omega_{2} \omega_{3} \ldots\right)=\omega_{2} \omega_{3} \ldots$, and $\sigma^{n}$ denotes the $n^{\text {th }}$ iterate of $\sigma$. Then a self-similar structure is said to be post critically finite (p.c.f. for short) if $\mathscr{P}$ is a finite set. Further, $K$ equipped with this structure is called a p.c.f. self-similar set.

The nested fractals introduced by Lindstrøm [Li] are p.c.f. self-similar sets. For further examples of p.c.f. self-similar sets, we refer to Sect. 8 in [Ki2], as well as to Sect. 3 below. Hereafter, we fix a p.c.f. self-similar structure $\left(K, S,\left\{F_{\imath}\right\}_{\imath=1}^{N}\right)$. Also we assume that $K$ is connected, because oterhwise, $K$ is totally disconnected and there is no non-trivial diffusion (with continuous paths) on $K$. By Hata [Ha], a necessary and sufficient condition for $K$ to be connected is that for each pair $(i, j)$, there exist $i_{1}, i_{2}, \ldots, i_{n} \in\{1,2, \ldots, N\}$ such that $i_{1}=i, i_{n}=j$ and $F_{\imath_{m}}(K) \cap F_{\imath_{m+1}}(K) \neq \emptyset$ for $m=1,2, \ldots, n-1$.

The next topic is a calculus on a self-similar structure, including Dirichlet forms, Laplacians and Green's function.

Definition 1.3. $V_{0}=\pi(\mathscr{P})$ and for $m \geq 0$,

$$
V_{m}=\bigcup_{w \in W_{m}} F_{w}(\pi(\mathscr{P}))
$$

The finite sets $V_{m}$ are thought to be an approximating sequence of $K$. In fact it is easy to see that $V_{m} \subset V_{m+1}$ and $K=$ the closure of $V_{*}$, where $V_{*}=\bigcup_{m \geq 0} V_{m}$. In
particular, $V_{0}$ is thought of as the "boundary" of $K$. Remark. In the case of nested fractals, $V_{0}$ coincides with the set of essential fixed points of $K$.

Constructing, first, a calculus on a finite set $V_{m}$, we will then obtain a calculus on $K$ through the natural limit as $m \rightarrow \infty$.

Notation. Let $U$ and $V$ be sets.

(1) $l(V)=\{f \mid f: V \rightarrow \mathbb{R}\}$. We use $(f)_{p}$ or $f_{p}$ to denote the value of $f \in l(V)$ at $p \in V$. For $p \in V, \chi_{p} \in l(V)$ is defined by

$$
\chi_{p}(q)= \begin{cases}1 & \text { if } q=p \\ 0 & \text { otherwise. }\end{cases}
$$

(2) Let $A: l(V) \rightarrow l(U)$ be linear, then we use $A_{p q}$ or $(A)_{p q}$ to denote the value $\left(A \chi_{q}\right)_{p}$ for $q \in V$ and $p \in U$. Note that $\sum_{q \in V} A_{p q} f_{q}=(A f)_{p}$.

(3) $C(K)=\{f \mid f \in l(K)$ and $f$ is continuous on $K\}$. The space $C(K)$ is endowed with the metric of uniform convergence on $K$. Note that it can be viewed as a subset of $l\left(V_{*}\right)$ because the countably infinite set $V_{*}$ is dense in $K$.

Now we introduce a class of finite-difference operators on $V_{0}$, which are discrete Laplacians on the finite (non-empty) set $V_{0}$.

Definition 1.4. Let $\mathscr{H}\left(V_{0}\right)$ be a collection of linear operators $D$ from $l\left(V_{0}\right)$ to itself such that

(1) ${ }^{\iota} D=D$, 
(2) $D$ is irreducible; that is, for each $(p, q) \in V_{0} \times V_{0}$, there exists a sequence $\left\{p_{i}\right\}_{\imath=1}^{n}$ with $p_{1}=p, p_{n}=q$ and $D_{p_{2} p_{i+1}} \neq 0$ for all $i=1,2, \ldots, n$,

(3) $D_{p p}<0$ and $\sum_{q \in V_{0}} D_{p q}=0$ for all $p \in V_{0}$,

(4) $D_{p q} \geq 0$ if $p \neq q$.

$D \in \mathscr{H}\left(V_{0}\right)$ induces finite-difference operators $H_{m}: l\left(V_{m}\right) \rightarrow l\left(V_{m}\right)$ as follows, where $r=\left(r_{1}, r_{2}, \ldots, r_{N}\right)$ is thought of as representing a ratio of weights of the subsets $\left(F_{1}(K), F_{2}(K), \ldots, F_{N}(K)\right)$. Note that for $i=1,2, \ldots, N$, the map $F_{\imath}: K \rightarrow F_{i}(K)$ is a homeomorphism; however, $F_{\imath}(K)$ and $F_{j}(K)$ may have different "sizes."

Definition 1.5. Let $D \in \mathscr{H}\left(V_{0}\right)$ and let $r=\left(r_{1}, r_{2}, \ldots, r_{N}\right)$ with each $r_{i}>0$. We define a linear operator $H_{m}: l\left(V_{m}\right) \rightarrow l\left(V_{m}\right)$ by

$$
H_{m}=\sum_{w \in W_{m}} r_{w}^{-1 t} R_{w} D R_{w}
$$

where $R_{w}: l\left(V_{m}\right) \rightarrow l\left(V_{0}\right)$ is the natural restriction of $f \in l\left(V_{m}\right)$ to $F_{w}\left(V_{0}\right)$; that is, $R_{w}(f)=f \circ F_{w}$ and $r_{w}=r_{w_{1}} r_{w_{2}} \ldots r_{w_{m}}$ for $w=w_{1} w_{2} \ldots w_{m}$. Moreover, we write $H_{m, p} f=\left(H_{m} f\right)_{p}$ for $p \in V_{m}$.

For further arguments, it is important that the pair $(D, r)$ be assumed to be invariant under a kind of "renormalization." To introduce the desired "renormalization," we divide $H_{m}$ into four parts as follows:

$$
H_{m}=\left(\begin{array}{cc}
T_{m} & { }^{t} J_{m} \\
J_{m} & X_{m}
\end{array}\right),
$$

where $T_{m}: l\left(V_{0}\right) \rightarrow l\left(V_{0}\right), J_{m}: l\left(V_{0}\right) \rightarrow l\left(V_{m} \backslash V_{0}\right)$ and $X_{m}: l\left(V_{m} \backslash V_{0}\right) \rightarrow l\left(V_{m} \backslash V_{0}\right)$.

Definition 1.6. $(D, r) \in \mathscr{H}\left(V_{0}\right) \times(0, \infty)^{N}$ is called a harmonic structure if

$$
D=\lambda\left(T-{ }^{t} J X^{-1} J\right)
$$

for some positive $\lambda>0$, where $T=T_{1}, X=X_{1}$ and $J=J_{1}$. Further, if $\lambda>r_{i}$ for $i=1,2, \ldots, N$, then $(D, r)$ is said to be a regular harmonic structure.

Remark. Let $\mathscr{S}_{r}(D)=T-{ }^{t} J X^{-1} J$, then $\mathscr{S}_{r}: \mathscr{H}\left(V_{0}\right) \rightarrow \mathscr{H}\left(V_{0}\right)$. Hence the above definition of harmonic structure is equivalent to the conditon that $D$ is an eigenfunction of the non-linear operator $\mathscr{S}_{r}$ with a positive eigenvalue. This operator $\mathscr{S}_{r}$ is a kind of renormalization of finite-difference operators on $V_{0}$. Essentially the same renormalization equations have been considered by Hattori-Hattori-Watanabe [HHW], Lindstrøm [Li] and Kusuoka [Ku2] in other approaches.

From now on, we focus our attention on a regular harmonic structure $(D, r)$ associated to a p.c.f. self-similar structure of $K$. It is natural to ask whether there always exists a regular harmonic structure. Unfortunately, a general answer to this question has not yet been found. For the nested fractals, however, there exists a regular harmonic structure with equal ratio of weights $r=(1,1, \ldots, 1)$. This result is obtained (with a different terminology) by Lindstrøm in [Li]. See [Ki2] and Sect. 3 below for several concrete examples of regular harmonic structures. 
The natural discrete Dirichlet form associated with $H_{m}$ is given by

$$
\mathscr{E}_{m}(u, v)=\lambda^{m t} u H_{m} v
$$

for $u, v \in l\left(V_{m}\right)$. By virtue of the invariance under renormalization, we have

Proposition 1.7. Let

$$
\mathscr{F}=\left\{u \mid u \in l\left(V_{*}\right), \mathscr{E}_{m}\left(\left.u\right|_{V_{m}},\left.u\right|_{V_{m}}\right) \text { has a finite limit as } m \rightarrow \infty\right\},
$$

where $\left.u\right|_{V_{m}}$ denotes the restriction of the function $u$ to $V_{m}$.

Then $\mathscr{F}$ is a dense subspace of $C(K)$. Moreover, for $u, v \in \mathscr{F}$, let

$$
\mathscr{E}(u, v)=\lim _{m \rightarrow \infty} \mathscr{E}_{m}\left(\left.u\right|_{V_{m}},\left.v\right|_{V_{m}}\right)
$$

and

$$
\overline{\mathscr{E}}(u, v)=\mathscr{E}(u, v)+\sum_{p \in V_{0}} u(p) v(p)
$$

Then $(\mathscr{F}, \overline{\mathscr{E}})$ is a Hilbert space.

Next we define the harmonic functions associated with $(\mathscr{E}, \mathscr{F})$.

Proposition 1.8. A continuous function $f$ on $K$ is called harmonic if $H_{m, p} f=0$ for all $m \geq 1$ and all $p \in V_{m} \backslash V_{0}$. Then

(1) The space of harmonic functions is contained in $\mathscr{F}$.

(2) For any $\varrho \in l\left(V_{0}\right)$, there exists a unique harmonic function $f$ such that $\left.f\right|_{V_{0}}=\varrho$. Moreover, $f$ is also the unique solution of the following variational problem:

$$
\mathscr{E}(f, f)=\min \left\{\mathscr{E}(u, u) \mid u \in \mathscr{F} \text { and }\left.u\right|_{V_{0}}=\varrho\right\} .
$$

Furthermore, for $p \in V_{0}$, we denote by $\psi_{p}$ a harmonic function whose values on $V_{0}$ coincide with $\chi_{p}$.

Also, Green's function $g$ is defined as follows.

Proposition 1.9. There exists a unique continuous function $g$ on $K \times K$ such that for all $x \in K$ and for every $f \in \mathscr{F}$,

$$
\mathscr{E}\left(g^{x}, f\right)=f(x)-\sum_{p \in V_{0}} f(p) \psi_{p}(x),
$$

where $g^{x}$ is defined by $g^{x}(y)=g(x, y)$. Further, $g$ is a non-negative valued function, $g^{p} \equiv 0$ for all $p \in l\left(V_{0}\right)$ and $g(x, y)=g(y, x)$ for all $x, y \in K$.

Note that all the concepts introduced so far are independent of the choice of a measure on $K$. To define the Laplace operators, however, we will need to introduce a measure on $K$. Hence, from now on, we will fix a (Borel) probability measure $\mu$ on $K$ such that

(1) $\mu(O)>0$ for all non-empty open sets $O$ in $K$.

(2) $\mu$ is non-atomic; that is, $\mu(U)=0$ for every finite set $U$.

A familiar example of a measure satisfying the above conditions is the Bernoulli measure $\mu$ which is characterized by

$$
\mu\left(K_{w}\right)=\mu_{w_{1}} \mu_{w_{2}} \ldots \mu_{w_{m}}
$$


for all $w=w_{1} w_{2} \ldots w_{m}$, where $\mu_{1}, \mu_{2}, \ldots, \mu_{N}$ are positive numbers such that $\sum_{i=1}^{N} \mu_{i}=1$. (Note that $\mu_{\imath}=\mu\left(F_{i}(K)\right)$, for $i=1, \ldots, N$.)

We need one more remark before giving the definition of Laplacians. For $p \in V_{m}$, we define $\psi_{m, p}$ as the unique continuous function on $K$ such that

(1) $\left.\psi_{m, p}\right|_{V_{m}}=\chi_{p}$,

(2) $\psi_{m, p} \circ F_{w}$ is harmonic for all $w \in W_{m}$.

Obviously, $\left\{\psi_{m, p}\right\}_{p \in V_{m}}$ is a partition of unity on $K$ and if $p \notin K_{w}$, then $\psi_{m, p} \equiv 0$ on $K_{w}$ for all $w \in W_{m}$. Hence, if we set $\mu_{m}=\sum_{p \in V_{m}} \mu_{m, p} \delta_{p}$, where $\mu_{m, p}=\int_{K} \psi_{m, p} d \mu$ and $\delta_{p}$ denotes Dirac's measure at $p$, then $\mu_{m}$ converges weakly to $\mu$ as $m \rightarrow \infty$. And so $\mu_{m}$ on $V_{m}$ can be thought of as a discrete approximation of $\mu$ on $K$.

Definition 1.10. Let $\mu$ be a probability measure satisfying the above conditions. For $u \in C(K)$, if there exists $f \in C(K)$ such that, as $m \rightarrow \infty$,

$$
\sup _{p \in V_{m} \backslash V_{0}}\left|\left(\Delta_{\mu}^{m} u\right)_{p}-f(p)\right| \rightarrow 0,
$$

where $\left(\Delta_{\mu}^{m} u\right)_{p}=\lambda^{m} \mu_{m, p}^{-1} H_{m, p} u$, then we define $\Delta_{\mu} u$ by $\Delta_{\mu} u=f$. The domain of $\Delta_{\mu}$ is denoted by $\mathscr{V}_{\mu}$. Further, the operator $\Delta_{\mu}$ is called the Laplacian on $K$ associated with $\mu$.

Remark 1. Naturally, $\Delta_{\mu}$ depends not only on the measure $\mu$ but also on the given harmonic and self-similar structures on $K$.

Remark 2. Clearly, $\Delta_{\mu}$ is a local operator, in the sense that (for $f \in \mathscr{D}_{\mu}$ and $x_{0} \in K$ ) if $f \equiv 0$ near $x_{0}$, then so does $\Delta_{\mu} f$.

Proposition 1.11. $(\mathscr{E}, \mathscr{F})$ is a local regular Dirichlet form on $L^{2}(K, \mu)$ and $\mathscr{D}_{\mu} \subset$ $\mathscr{F} \subset C(K)$.

For the definitions and fundamental results on Dirichlet forms, we refer to Fukushima [Fu1]. (See also Definition 4.1 below where is recalled the definition of a Dirichlet form.)

Finally we state two results that are analogues of classical facts for the ordinary Laplacian, Green's function and Dirichlet form on ("smooth" bounded domains of) $\mathbb{R}^{n}$. At first, we give a formula for the solution of the Dirichlet problem for Poisson's equation.

Theorem 1.12. Given $f \in C(K)$ and $\varrho \in l\left(V_{0}\right)$, there exists a unique $u \in \mathscr{F}$ such that

$$
\left\{\begin{array}{l}
\Delta_{\mu} u=f \\
\left.u\right|_{V_{0}}=\varrho
\end{array}\right.
$$

Moreover, this function $u$ is given by

$$
u(x)=\sum_{p \in V_{0}} \varrho(p) \psi_{p}(x)-\int_{K} g(x, y) f(y) \mu(d y) .
$$

In particular, $v \in C(K)$ is harmonic if and only if $v \in \mathscr{D}_{\mu}$ and $\Delta_{\mu} v=0$. 
The second result is the counterpart of "Gauss-Green's formula" in this context.

Theorem 1.13. For $u \in \mathscr{F}$ and $v \in \mathscr{D}_{\mu}$, we have

$$
\mathscr{E}(u, v)=\sum_{p \in V_{0}} u(p)(d v)_{p}-\int_{K} u \Delta_{\mu} v d \mu,
$$

where $(d v)_{p}$ is called the Neumann derivative of $v$ at $p \in V_{0}$ and is defined by

$$
(d v)_{p}=\lim _{m \rightarrow \infty}-\lambda^{m} H_{m, p} u .
$$

The above limit does exist if $v \in \mathscr{D}_{\mu}$.

\section{Main Results; the Spectrum of Laplacians}

In this section, we state and prove our main results about the asymptotic distribution of the spectrum of the Laplacians defined in Sect. 1. We fix a p.c.f. self-similar structure $\left(K, S,\left\{F_{i}\right\}_{i=1}^{N}\right)$ and a regular harmonic structure $(D, r)$ on this p.c.f. selfsimilar structure. Further, $\mu$ is an arbitrary Bernoulli measure on $K$ (as defined after Proposition 1.9). Under these conditions, the Laplacian $\Delta_{\mu}$ acts from $\mathscr{D}_{\mu}$ to $C(K)$. For simplicity, we will write $\mathscr{D}$ and $\Delta$ instead of $\mathscr{D}_{\mu}$ and $\Delta_{\mu}$, respectively.

First we formulate the eigenvalue problem of $-\Delta$ with (homogeneous) Neumann boundary conditions and Dirichlet boundary conditions (for short, the Neumann and Dirichlet Laplacians).

Definiton 2.1. For $k \in \mathbb{R}$ and $u \in \mathscr{Q}$, if

$$
\left\{\begin{array}{l}
\Delta u=-k u, \\
(d u)_{p}=0 \text { for all } p \in V_{0},
\end{array}\right.
$$

then $k$ is said to be an eigenvalue of $-\Delta$ with Neumann boundary conditions and $u$ is said to be an associated eigenfunction. Also, if

$$
\left\{\begin{array}{l}
\Delta u=-k u \\
\left.u\right|_{V_{0}}=0
\end{array}\right.
$$

then $k$ is said to be an eigenvalue of $-\Delta$ with Dirichlet boundary conditions and $u$ is said to be an associated eigenfunction.

As we will see in Sect. 4 , the eigenvalues of $-\Delta$ are non-negative and have finite multiplicity and they have no limit point other than $+\infty$ under either Dirichlet or Neumann boundary conditions. Hence we can consider the associated (eigenvalue) counting function.

Definition 2.2. The eigenvalue counting function of $-\Delta$ with Neumann boundary conditions is given by

$$
\varrho(x)=\#\left\{k / \begin{array}{l}
k \text { is an eigenvalue of }-\Delta \text { with Neumann } \\
\text { boundary conditions, with } k \leq x
\end{array}\right\},
$$

where each eigenvalue is counted according to its multiplictiy and $\# A$ denotes the number of elements in the (finite) set $A$.

Similarly, we define $\varrho_{0}(x)$, the eigenvalue counting function of $-\Delta$ with Dirichlet boundary conditions. 
Obviously, $\varrho(x)$ and $\varrho_{0}(x)$ are non-decreasing and right continuous. Further, note that $\varrho(0)=1$, because 0 is the first Neumann eigenvalue (and $K$ is connected), whereas $\varrho_{0}(0)=0$. (See Sect. 4.) We are interested in the asymptotic behavior of $\varrho(x)$ and $\varrho_{0}(x)$ as $x \rightarrow \infty$. The following facts are crucial for obtaining our main results.

Lemma 2.3. For all $x \geq 0$, we have

$$
\sum_{i=1}^{N} \varrho_{0}\left(\frac{r_{i} \mu_{i}}{\lambda} x\right) \leq \varrho_{0}(x) \leq \varrho(x) \leq \sum_{i=1}^{N} \varrho\left(\frac{r_{i} \mu_{i}}{\lambda} x\right)
$$

and

$$
\varrho_{0}(x) \leq \varrho(x) \leq \varrho_{0}(x)+M
$$

where $M=\#\left(V_{0}\right)$.

The proof of part (1) (resp., (2)) of Lemma 2.3 will be given in Sect. 5 (resp., Sect. 6). Part (2) will be proven by using a suitable refinement of the method of "Dirichlet-Neumann bracketing" - as was done in [La1] for regions with fractal boundary - and by also taking into account the specific features of our present situation, particularly the finite-dimensionality of the space of harmonic functions on p.c.f. self-similar sets. Fukushima [Fu2] has obtained part (1) of Lemma 2.3 in the special case of nested fractals where $r_{i}=1$ and $\mu_{i}=\frac{1}{N}$ for all $i=1,2, \ldots, N$, so that $\mu$ is the natural Hausdorff measure on $K$.

We now present our main results, which provide an analogue of Weyl's theorem for Laplacians on p.c.f. self-similar fractals. Our result for the Dirichlet problem is stated in the following theorem, while the corresponding result for the Neumann problem is given in Corollary 2.5 below.

Theorem 2.4. Under the above hypotheses, we have

$$
0<\operatorname{liminv}_{x \rightarrow \infty} \varrho_{0}(x) x^{-\frac{d_{S}}{2}} \leq \limsup _{x \rightarrow \infty} \varrho_{0}(x) x^{-\frac{d_{S}}{2}}<\infty ;
$$

i.e., there exist constants $c_{1}, c_{2}>0$ such that $c_{1} x^{d_{S} / 2} \leq \varrho_{0}(x) \leq c_{2} x^{d_{S} / 2}$, for all $x$ large enough. Here, $d_{S}$ is the unique positive number such that

$$
\sum_{i=1}^{N}\left(\frac{r_{i} \mu_{i}}{\lambda}\right)^{\frac{d_{S}}{2}}=1
$$

Further, let $\gamma_{i}=\sqrt{\frac{r_{\imath} \mu_{\imath}}{\lambda}}$, for $i=1, \ldots, N$, so that $\sum_{i=1}^{N} \gamma_{i}^{d_{S}}=1$. Then (1) Non-Lattice Case: If the (additive) group $\sum_{i=1}^{N} \mathbb{Z} \log \gamma_{i}$ is a dense subgroup of $\mathbb{R}$,
then

$$
\lim _{x \rightarrow \infty} \varrho_{0}(x) x^{-\frac{d_{S}}{2}}=\left(-\sum_{i=1}^{N} \gamma_{\imath}^{d_{S}} \log \gamma_{i}\right)^{-1} \int_{-\infty}^{\infty} e^{-d_{S} t} R\left(e^{2 t}\right) d t
$$

where ( $R$ is bounded, right continuous and) $R(x):=\varrho_{0}(x)-\sum_{i=1}^{N} \varrho_{0}\left(\frac{r_{i} \mu_{i}}{\lambda} x\right)$. 


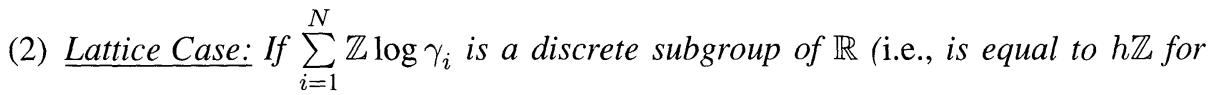
some $h>0$ ), let $T$ be its positive generator (i.e., the smallest such number $h>0$ ). Then

$$
\varrho_{0}(x)=\left(G\left(\frac{\log x}{2}\right)+o(1)\right) x^{\frac{d_{S}}{2}},
$$

where o(1) denotes a term which vanishes as $x \rightarrow \infty$ and $G$ is a positive periodic function with period $T$ given by

$$
G(t)=T\left(-\sum_{i=1}^{N} \gamma_{i}^{d_{S}} \log \gamma_{\imath}\right)^{-1} \sum_{j=-\infty}^{+\infty} e^{-d_{S}(t+\jmath T)} R\left(e^{2(t+j T)}\right) .
$$

Moreover, $G$ is right continuous and bounded from above and away from zero.

The next corollary follows immediately from Theorem 2.4 and part (2) of Lemma 2.3.

Corollary 2.5. Under the hypotheses of Theorem 2.4, exactly the same results hold for $\varrho(x)$, the eigenvalue counting function for the Neumann Laplacian. Moreover, $d_{S}$ is still given by (2.2) and, in the counterpart of (2.3)-(2.5), the functions $R$ and $G$ involved are the same as in the case of $\varrho_{0}(x)$.

We refer the reader to Sect. 3 (as well as to the appendix) for a discussion of several examples illustrating our main results.

Remark 1. In the original Weyl's theorem, the positive constant $\lim _{x \rightarrow \infty} \varrho_{0}(x) x^{-\frac{n}{2}}$ has a simple geometric interpretation; namely, it is expressed in terms of the volume of the domain in $\mathbb{R}^{n}$ (see Theorem 0.1 ). Unfortunately, we do not have a similar interpretation for the value of the limit in our non-lattice case. Actually, in view of the results and examples in [LP3] and [La3] obtained for regions with fractal boundary, it may not be easy to find one. Nevertheless, our present results show that in the non-lattice case, the proportionality "constant" involved in (2.3) is the same for the Dirichlet and Neumann problems (see Corollary 2.5).

Remark 2. In the lattice case and when $d_{S}$ is not an integer, we conjecture that the periodic function $G(t)$ in (2.4) is non-constant, and hence that the limit in (2.3) does not exist. Intuitively, these oscillations in the leading asymptotics of the spectrum should be caused by the high symmetry of $K$ (as well as of $(D, r)$ and $\mu$ ) which gives rise to eigenvalues with large multiplicity. (Compare [LP2, Remark 4.7(b), p. 67].) According to the result of Fukushima and Shima [FS], this conjecture is true for the standard Laplacian on the Sierpinski gasket (which, of course, corresponds to the lattice case since then, $r_{1}=\ldots=r_{N}=1$ and $\left.\mu_{1}=\ldots=\mu_{N}=\frac{1}{N}\right)$. It is noteworthy that when $d_{S}$ is an integer, the limit in (2.3) may exist even in the lattice case. For example, if $K$ is the Koch snowflake curve, equipped with its natural Hausdorff measure $\mu$, then $K$ is isomorphic to the unit segment $[0,1]$, equipped with Lebesgue measure; hence $d_{S}=1$ and the limit in (2.3) exists.

Remark 3. ("Spectral exponents".) In view of the above results, the positive number $d_{S}$ defined by (2.2) determines the asymptotic order of the eigenvalue distribution of the Laplacian. We will call $d_{S}$ "the spectral exponent of the Laplacian." It depends 
not only on the space $K$ but also on the self-similar structure $\left(K, S,\left\{F_{i}\right\}_{i=1}^{N}\right)$, the harmonic structure $(D, r)$ and the probability measure $\mu$ on $K$. In the case of the Sierpinski gasket, the spectral exponent of the standard Laplacian, $d_{S}=\ln 9 / \ln 5$, has been called "the spectral dimension of the Sierpinski gasket." As was noted above, however, there are many spectral exponents associated to a given p.c.f. self-similar structure. In an appendix to this paper, we shall propose a notion of spectral dimension corresponding to a specific choice of spectral exponent. (See Theorem A.2.) Of course, it will be equal to $\ln 9 / \ln 5$ in the case of the Sierpinski gasket.

Note that our (direct) definition of $d_{S}$ is very analogous to that of the "similarity dimension" of a (strictly) self-similar set (e.g., [Fa, Sect. 9.2, esp. Theorem 9.3, p. 118]); namely, $d_{S}$ is the positive solution of the equation

$$
\sum_{i=1}^{N} \gamma_{i}^{d_{S}}=1
$$

However, the coefficients $\gamma_{i}$ no longer represent the scaling factors of the similarity transformations involved, but rather are now defined by $\gamma_{i}=\left(\mu_{i} r_{\imath} / \lambda\right)^{1 / 2}$, for $i=2, \ldots, N$. Finally, observe that in our case, $d_{S}<2$ because $\sum_{i=1}^{N} \gamma_{i}^{2}<1$. This follows since $\left(\mu_{i} r_{i} / \lambda\right)<\mu_{i}$ and $\sum_{i=1}^{N} \mu_{i}=1$, where we use the fact that $r_{i}<\lambda$ because the harmonic structure is assumed to be regular, in the sense of Definition 1.6. Hence, in the present setting, $d_{S}$ is an integer if and only if $d_{S}=1$, which is noteworthy in view of Remark 2 above.

Remark 4. Combining ideas of Kusuoka [Ku2] and Fukushima [Fu2], Kumagai [Km1] has obtained the value of the "spectral exponent" for a special Bernoulli measure and under certain rather technical conditions on the (regular or non-regular) harmonic structure. After having learned about our present work, he then removed these technical conditions in $[\mathrm{Km} 2]$, still working with the above measure, and also remarked that our methods and his could be combined to extend our main results (Theorem 2.4 and Corollary 2.5 above) to non-regular structures, thereby allowing $d_{S}$ to be greater than 2 .

Remark 5. When $N=2$, the lattice case occurs if and only if $\log \gamma_{2} / \log \gamma_{1}$ is rational; i.e., if and only if $\gamma_{2}^{q}=\gamma_{1}^{p}$ for some positve integers $p$ and $q$. Clearly, a similar criterion holds when $N \geq 2$.

Proof of the Main Results. The rest of this section is devoted to the proof of Theorem 2.4. First, for a given integer $n \geq 1$, we define a collection of words with various length, $\Lambda_{n} \subset \bigcup_{m \geq 1} W_{m}$, by

$$
\Lambda_{n}=\left\{w=w_{1} w_{2} \ldots w_{m} \mid\left(\gamma_{w_{1} w_{2} \ldots w_{m-1}}\right)^{d_{S}}>\alpha^{n} \geq \gamma_{w}^{d_{S}}\right\}
$$

where $\alpha=\min \left\{\gamma_{1}^{d_{S}}, \gamma_{2}^{d_{S}}, \ldots, \gamma_{N}^{d_{S}}\right\}$ and $\gamma_{w}=\gamma_{w_{1}} \gamma_{w_{2}} \ldots \gamma_{w_{m}}$. (An analogous set was introduced by Moran in [Mo].) The following facts are easily deduced from the definition.

\section{Lemma 2.6.}

$$
\alpha^{n} \geq \gamma_{w}^{d_{S}}>\alpha^{n+1} \quad \text { for all } w \in \Lambda_{n}
$$


and

$$
\bigcup_{w \in \Lambda_{n}} \Sigma_{w}=\Sigma \quad \text { and } \quad \Sigma_{w} \cap \Sigma_{w^{\prime}}=\emptyset \quad \text { for } \quad w, w^{\prime} \in \Lambda_{n} \quad \text { with } w \neq w^{\prime}
$$

where $\Sigma_{w}=\left\{\omega \in \Sigma \mid \omega=w \omega_{k} \omega_{k+1} \ldots\right\}$.

Lemma 2.7. Let $\Lambda \subset \bigcup_{m \geq 1} W_{m}$ satisfying $\bigcup_{w \in \Lambda} \Sigma_{w}=\Sigma$ and $\Sigma_{w} \cap \Sigma_{w^{\prime}}=\emptyset$ for $w, w^{\prime} \in \Lambda$ with $w \neq w^{\prime}$. Then given $f:[0, \infty) \rightarrow \mathbb{R}$ and positive constants $\alpha_{1}, \alpha_{2}, \ldots, \alpha_{N}$, we have:

$$
\text { If } f(x) \geq \sum_{i=1}^{N} f\left(\alpha_{i} x\right), \text { then } f(x) \geq \sum_{w \in \Lambda} f\left(\alpha_{w} x\right),
$$

where $\alpha_{w}=\alpha_{w_{1}} \alpha_{w_{2}} \ldots \alpha_{w_{m}}$ for $w=w_{1} w_{2} \ldots w_{m}$.

$$
\begin{aligned}
& \text { If } f(x)=\sum_{i=1}^{N} f\left(\alpha_{i} x\right), \text { then } f(x)=\sum_{w \in \Lambda} f\left(\alpha_{w} x\right) . \\
& \text { If } f(x) \leq \sum_{i=1}^{N} f\left(\alpha_{i} x\right), \text { then } f(x) \leq \sum_{w \in \Lambda} f\left(\alpha_{w} x\right) .
\end{aligned}
$$

Proof of Lemma 2.7. We will show (1). We define $n(\Lambda)=\min \left\{m \mid \Lambda \subset \bigcup_{i=1}^{m} W_{\imath}\right\}$ and use an induction on $n(\Lambda)$. First, if $n(\Lambda)=1$, then obviously $\Lambda=\{1,2, \ldots, N\}$ and so the claim holds. Next if $n=n(\Lambda)>1$, then, for $w=w_{1} w_{2} \ldots w_{n} \in W_{n} \cap \Lambda$, we can see that $\left\{w_{1} w_{2} \ldots w_{n-1} j\right\}_{j=1}^{N} \subset \Lambda$. Further, we note that the assumption implies

$$
f\left(\alpha_{w_{1} w_{2} \ldots w_{n-1}} x\right) \geq \sum_{j=1}^{N} f\left(\alpha_{w_{1} w_{2} \ldots w_{n-1} j} x\right) .
$$

And so, if we let

$$
\Lambda^{\prime}=\left(\Lambda \cap\left(\bigcup_{i=1}^{n-1} W_{i}\right)\right) \cup\left\{w_{1} w_{2} \ldots w_{n-1} \mid w=w_{1} w_{2} \ldots w_{n} \in W_{n} \cap \Lambda\right\},
$$

then it follows that $\Lambda^{\prime}$ satisfies the same conditions as $\Lambda, n\left(\Lambda^{\prime}\right)=n(\Lambda)-1$ and $\sum_{w \in \Lambda^{\prime}} f\left(\alpha_{w} x\right) \geq \sum_{w \in \Lambda} f\left(\alpha_{w} x\right)$. Hence by the induction hypothesis, we have $f(x) \geq \sum_{w \in \Lambda} f\left(\alpha_{w} x\right)$

The proof of (2) and (3) is entirely similar.

If we now combine Lemma 2.6 and Lemma 2.7, Lemma 2.3-(1) implies

$$
\sum_{w \in \Lambda_{n}} \varrho_{0}\left(\gamma_{w}^{2} t\right) \leq \varrho_{0}(t) \leq \varrho(t) \leq \sum_{w \in \Lambda_{n}} \varrho\left(\gamma_{w}^{2} t\right)
$$

Since $\varrho_{0}$ and $\varrho$ are non-decreasing, we have

$$
\#\left(\Lambda_{n}\right) \varrho_{0}\left(\alpha^{(n+1) \frac{2}{d_{S}}} t\right) \leq \varrho_{0}(t) \leq \varrho(t) \leq \#\left(\Lambda_{n}\right) \varrho\left(\alpha^{n \frac{2}{d_{S}} t}\right) .
$$


On the other hand, by Lemma 2.7-(2), we have

$$
\sum_{w \in \Lambda_{n}} \gamma_{w}^{d_{S}}=1 \quad \text { and this implies } \#\left(\Lambda_{n}\right) \alpha^{n} \geq 1 \geq \#\left(\Lambda_{n}\right) \alpha^{n+1} .
$$

Hence we have

$$
\alpha^{-n} \varrho_{0}\left(\alpha^{(n+1) \frac{2}{d_{S}}} t\right) \leq \varrho_{0}(t) \leq \varrho(t) \leq \alpha^{-(n+1)} \varrho_{0}\left(\alpha^{n \frac{2}{d_{S}}} t\right) .
$$

Replacing $t$ by $t \alpha^{-n \frac{2}{d_{S}}}$, we obtain

$$
\varrho_{0}\left(\alpha^{\frac{2}{d_{S}}} t\right) \leq \alpha^{n} \varrho_{0}\left(t \alpha^{-n \frac{2}{d_{S}}}\right) \leq \alpha^{n} \varrho\left(t \alpha^{-n \frac{2}{d_{S}}}\right) \leq \alpha^{-1} \varrho(t) .
$$

Now we choose $t$ so that $\varrho_{0}\left(\alpha^{\frac{2}{d_{S}} t}\right)>0$. For $x \in\left[t \alpha^{-n \frac{2}{d_{S}}}, t \alpha^{-(n+1) \frac{2}{d_{S}}}\right]$,

$$
t^{-\frac{d_{S}}{2}} \alpha^{n+1} \varrho_{0}\left(t \alpha^{-n \frac{2}{d_{S}}}\right) \leq \varrho_{0}(x) x^{-\frac{d_{S}}{2}} \leq t^{-\frac{d_{S}}{2}} \alpha^{n} \varrho_{0}\left(t \alpha^{-(n+1) \frac{2}{d_{S}}}\right) .
$$

Therefore, we have

$$
t^{-\frac{d_{S}}{2}} \alpha \varrho_{0}\left(\alpha^{\frac{2}{d_{S}}} t\right) \leq \varrho_{0}(x) x^{-\frac{d_{S}}{2}} \leq t^{-\frac{d_{S}}{2}} \alpha^{-2} \varrho(t) .
$$

This implies immediately (2.1) from Theorem 2.4:

$$
0<\operatorname{liminv}_{x \rightarrow \infty} \varrho_{0}(x) x^{-\frac{d_{S}}{2}} \leq \limsup _{x \rightarrow \infty} \varrho_{0}(x) x^{-\frac{d_{S}}{2}}<\infty .
$$

To establish the refined results stated in Theorem 2.4, we need the following wellknown renewal theorem from probability theory.

The Renewal Theorem (Feller [Fe]). Let $\nu$ be a Borel probability measure on $[0, \infty)$ such that $\int_{0}^{\infty} x \nu(d x)<\infty$. Let $u \in L^{1}(\mathbb{R})$ be such that $u(x) \rightarrow 0$ as $|x| \rightarrow \infty$. Suppose that $z$ is a bounded measurable function which satisfies the renewal equation

$$
z(x)=u(x)+\int_{0}^{\infty} z(x-t) \nu(d t), \quad \text { for } x \in \mathbb{R},
$$

and such that $z(x) \rightarrow 0$ as $x \rightarrow-\infty$.

Then

(1) Non-Lattice Case: If the support of $\nu$ does not lie in any discrete subgroup of $\mathbb{R}$, then the limit $z(\infty)=\lim _{x \rightarrow \infty} z(x)$ exists and

$$
z(\infty)=\left(\int_{0}^{\infty} x \nu(d x)\right)^{-1} \int_{-\infty}^{+\infty} u(x) d x .
$$

(2) Lattice Case: If the support of $\nu$ lies in some discrete subgroup of $\mathbb{R}$, then if $T$ is the "greatest common divisor" of the support of $\nu$, the limit $G(t)=\lim _{n \rightarrow \infty} z(t+n T)$ exists for every $t$ and

$$
G(t)=\left(\int_{0}^{\infty} x \nu(d x)\right)^{-1} \sum_{j=-\infty}^{+\infty} u(t+j T) .
$$


Remark 1. The above version of the renewal theorem is a slight modification of the classical renewal theorem [Fe, Theorem 2.1, p. 349], which follows easily from the proof given in [Fe] as well as from [Ru, Theorem 9.15, pp. 219-220]. Note that in [Fe], the "lattice case" is called the "arithmetic case.")

Remark 2. Lalley [L] has used the renewal theorem in a related context in order to study the asymptotic behavior of certain geometric counting functions associated with self-similar fractals in $\mathbb{R}^{n}$.

Lemma 2.8. Let $R(x)=\varrho_{0}(x)-\sum_{i=1}^{N} \varrho_{0}\left(\gamma_{i}^{2} x\right)$, where $\gamma_{i}=\left(r_{i} \mu_{i} / \lambda\right)^{1 / 2}$; then $R(x)$ is a non-negative bounded function and, for some $k_{1}>0, R(x)=0$ on $\left[0, k_{1}\right]$.

Proof of Lemma 2.8. By Lemma 2.3-(1), $R(x)$ is non-negative. Also by Lemma 2.3,

$$
\varrho_{0}(x) \leq \sum_{i=1}^{N} \varrho\left(\gamma_{i}^{2} x\right) \leq \sum_{i=1}^{N}\left(\varrho_{0}\left(\gamma_{i}^{2} x\right)+M\right) .
$$

Hence $R(x) \leq M N$. Finally, let $k_{1}$ be the smallest eigenvalue of $-\Delta$ with Dirichlet boundary conditions. Then it is easy to show that $k_{1}>0$. (See Sect. 4.) Therefore $\varrho_{0}\left(\gamma_{i}^{2} x\right)=0\left(\right.$ since $\left.\gamma_{i}<1\right)$ and hence $\varrho_{0}(x)=0$ on $\left[0, k_{1}\right]$. This implies that $R(x)=0$ on $\left[0, k_{1}\right]$.

End of the proof of Theorem 2.4 . We let $z(t)=e^{-t d_{S}} \varrho_{0}\left(e^{2 t}\right), u(t)=e^{-t d_{S}} R\left(e^{2 t}\right)$ and $\nu(d t)=\sum_{i=1}^{N} \gamma_{i}^{d_{S}} \delta_{-\log \gamma_{2}}(d t)$, where $\delta_{x}$ is the Dirac point mass at $x$. (Observe that $\nu$ is a probability measure since by definition of $d_{S}$, we have $\sum_{i=1}^{N} \gamma_{i}^{d_{S}}=1$; see (2.2) or (2.6). Then, using (2.6) again, we immediately deduce the renewal equation (2.7):

$$
z(x)=u(x)+\int_{0}^{\infty} z(x-t) \nu(d t) .
$$

Further, it is easy to check that the assumptions of the above renewal theorem are satisfied. Thus Theorem 2.4 is now a direct consequence of the renewal theorem.

\section{Examples}

This section is devoted to examples illustrating our results. In each example, item (I) describes the self-similar structure, item (II) describes harmonic structures and in item (III), we discuss various properties of the spectral exponents of Laplacians. In the appendix, we shall revisit several of these examples from the point of view of the spectral dimension.

Example 1. Interval

(I) For $i=1,2$, the maps $F_{\imath}: \mathbb{R} \rightarrow \mathbb{R}$ are contractions defined by

$$
F_{1}(x)=\frac{1}{2} x \quad \text { and } \quad F_{2}(x)=\frac{1}{2} x+\frac{1}{2} .
$$

Let $K=[0,1]$. Then $\left(K,\{1,2\},\left\{F_{1}, F_{2}\right\}\right)$ is a p.c.f. self-similar structure. In fact,

$$
\begin{gathered}
\mathscr{C}=\{1 \dot{2}, 2 \dot{1}\}, \quad \frac{1}{2}=\pi(1 \dot{2})=\pi(2 \dot{1}), \\
\mathscr{P}=\{\dot{1}, \dot{2}\}, \quad 0=\pi(\dot{1}) \quad \text { and } \quad 1=\pi(\dot{2}) .
\end{gathered}
$$


[Here and thereafter, we use the following convention. Given a symbol $s$, we denote by $\dot{s}$ the constant infinite sequence associated with this symbol $s$. For example, $i=1111 \ldots .$.

(II)

$$
D=\left(\begin{array}{cc}
-1 & 1 \\
1 & -1
\end{array}\right), \quad r=(\alpha, 1-\alpha)
$$

with $0<\alpha<1$. Then $(D, r)$ is a regular harmonic structure where $\lambda=1$.

(III) For a Bernoulli measure $\mu$, let $\mu_{1}=\beta$ and $\mu_{2}=1-\beta$ with $0<\beta<1$. The spectral exponent $d_{S}$ of the Laplacian given by $(D, r)$ and $\mu$ is characterized by

$$
(\alpha \beta)^{\frac{d_{S}}{2}}+((1-\alpha)(1-\beta))^{\frac{d_{S}}{2}}=1 .
$$

Consequently, $d_{S}=1$ if and only if $\alpha=\beta$, and $d_{S}<1$ otherwise. Further, we are in the lattice case if and only if $\log ((1-\alpha)(1-\beta)) / \log (\alpha \beta)$ is rational.

\section{Example 2. Sierpinski Gasket (Fig. 1)}

(I) Given an integer $N \geq 2$, consider the standard unit $N$-simplex in $\mathbb{R}^{N-1}$, with vertices $\left\{p_{1}, \ldots, p_{N}\right\}$ and edges of length 1 . For $i=1,2, \ldots, N, F_{i}: \mathbb{R}^{N-1} \rightarrow$ $\mathbb{R}^{N-1}$ is a contraction defined by

$$
F_{i}(x)=\frac{1}{2}\left(x-p_{i}\right)+p_{\imath} .
$$

The $N$-Sierpinski gasket is the unique (non-empty) compact set satisfying

$$
K=\bigcup_{i=1}^{N} F_{\imath}(K)
$$

Then $\left(K,\{1,2, \ldots, N\},\left\{F_{i}\right\}_{i=1,2, \ldots, N}\right)$ is a p.c.f. self-similar structure. In fact,

$$
\begin{gathered}
\mathscr{C}=\bigcup_{k<l}\{k \dot{l}, l \dot{k}\}, \quad \frac{1}{2}\left(p_{k} p_{l}\right)=\pi(k \dot{l})=\pi(l \dot{k}), \\
\mathscr{P}=\{\dot{1}, \dot{2}, \ldots, \dot{N}\} \quad \text { and } \quad p_{k}=\pi(\dot{k}) \quad \text { for } \quad k=1,2, \ldots, N,
\end{gathered}
$$

where $\frac{1}{2}\left(p_{k} p_{l}\right)$ denotes the midpoint of $p_{k}$ and $p_{l}$.

(II)

$$
D=\left(\begin{array}{cccc}
-(N-1) & 1 & \cdots & 1 \\
1 & -(N-1) & \ddots & \vdots \\
\vdots & \ddots & \ddots & \vdots \\
1 & \ldots & 1 & -(N-1)
\end{array}\right), \quad r=(1,1, \ldots, 1)
$$

Then $(D, r)$ is a regular harmonic structure where $\lambda=\frac{N+2}{N}$.

[For $N=2, K$ is an interval as in Example 1 above, whereas for $N=3, K$ is the S.G. discussed in the introduction and represented in Fig. 1b. Note that in Example 1, we chose $r=(1 / 2,1 / 2)$ whereas in the present case, we take $r=(1,1)$; this explains why here $\lambda=2$ instead of $\lambda=1$.] 
(III) The spectral exponent $d_{S}$ of the Laplacian given by $(D, r)$ and a Bernoulli measure $\mu$ is characterized by

$$
\left(\frac{N}{N+2}\right)^{\frac{d_{S}}{2}} \sum_{i=1}^{N} \mu_{i}^{\frac{d_{S}}{2}}=1
$$

We deduce from (3.2) that $d_{S} \leq 2 \log N / \log (N+2)$ and the equality holds if and only if $\mu$ is the normalized Hausdorff measure where $\mu_{\imath}=\frac{1}{N}$ for all $i=1,2, \ldots, N$. This latter situation, that of ordinary $N$-Sierpinski gasket, is that studied by FukushimaShima in [FS]; clearly, it corresponds to the "lattice case" (in our present terminology) and hence according to Theorem 2.4 and Corollary 2.5, for the associated Dirichlet and Neumann Laplacians, not only does (2.1) holds - as was already shown in [FS] - but also (2.4) holds, where the periodic function $G$ defined by (2.5) is non-constant since by [FS], $x^{-d_{S} / 2} \varrho(x)$ does not converge as $x \rightarrow \infty$. This value, $2 \log N / \log (N+2)$, will be called "the spectral dimension" in the appendix.

It is noteworthy that the Hausdorff dimension of $K$ is equal to $\log N / \log 2$ and that for $N>2$,

$$
\frac{2 \log N}{\log (N+2)}<\frac{\log N}{\log 2}
$$

Example 3. Modified Sierpinski Gasket (Fig. 2)

(I) Let $\left\{p_{1}, p_{2}, p_{3}\right\}$ be the vertices of a regular triangle in $\mathbb{C}$ and let

$$
p_{4}=\frac{1}{2}\left(p_{2} p_{3}\right), \quad p_{5}=\frac{1}{2}\left(p_{1} p_{2}\right), \quad p_{6}=\frac{1}{2}\left(p_{2} p_{3}\right) .
$$

Further, let the contraction $F_{\imath}: \mathbb{C} \rightarrow \mathbb{C}$ be defined by

$$
F_{i}(z)= \begin{cases}\alpha\left(z-p_{i}\right)+p_{\imath} & \text { for } i=1,2,3 \\ (1-2 \alpha)\left(z-p_{\imath}\right)+p_{i} & \text { for } i=4,5,6\end{cases}
$$

where $z \in \mathbb{C}$ and the parameter $\alpha$ satisfies $\frac{1}{3}<\alpha<\frac{1}{2}$. The unique compact set $K \subset \mathbb{C}$ which satisfies $K=\bigcup_{i=1}^{6} F_{\imath}(K)$ is called the modified Sierpinski gasket (M.S.G. for short). It is easy to check that $\left(K,\{1,2, \ldots, 6\},\left\{F_{i}\right\}_{\imath=1,2, \ldots, 6}\right)$ is a p.c.f. self-similar structure. In fact,

$$
\mathscr{C}=\bigcup_{\substack{k<l<m \\ k+l+m=9}}\{k \dot{l}, m \dot{k}, m \dot{l}, l \dot{k}\}, \quad q_{k m}=\pi(k \dot{l})=\pi(m \dot{k})
$$

and $q_{l m}=\pi(m \dot{l})=\pi(l \dot{k})$ for each triple $(k, l, m)$ such that $k<l<m$ and $k+l+m=9$. Also, $\mathscr{P}=\{\dot{1}, \dot{2}, \dot{3}\}$ and $p_{k}=\pi(\dot{k})$ for $k=1,2,3$.

The Hausdorff dimension of the M.S.G., denoted by $d_{H}$, is the unique positive number which satisfies

$$
3 \alpha^{d_{H}}+3(1-2 \alpha)^{d_{H}}=1 \text {. }
$$

$$
D=\left(\begin{array}{ccc}
-2 & 1 & 1 \\
1 & -2 & 1 \\
1 & 1 & -2
\end{array}\right), \quad r=(1,1,1, t, t, t)
$$


with $t>0$. Then $(D, r)$ is a harmonic structure and it is regular if and only if $0<t<\frac{-1+\sqrt{21}}{2}$.

(III) Of course, we could choose any Bernoulli measure $\mu$ on $K$. However, we now focus on the normalized Hausdorff measure, where $\mu_{1}=\mu_{2}=\mu_{3}=\alpha^{d_{H}}$ and $\mu_{4}=\mu_{5}=\mu_{5}=(1-2 \alpha)^{d_{H}}$, because this measure seems natural from the geometrical point of view. Then the spectral exponent $d_{S}$ of the Laplacian given by $(D, r)$ and $\mu$ is characterized by

$$
3\left(\frac{\alpha^{d_{H}}}{\lambda}\right)^{\frac{d_{S}}{2}}+3\left(\frac{t(1-2 \alpha)^{d_{H}}}{\lambda}\right)^{\frac{d_{S}}{2}}=1 .
$$

It is remarkable that there are no particular relations between $\alpha$ and $t$ and so these two parameters are mutually independent. In such a case, as was mentioned in Remark 3 of Theorem 2.4, we cannot use an expression such as "the spectral dimension of the M.S.G."

Finally, we note that for a fixed harmonic structure (i.e., for a fixed $t$ and hence $\lambda$ ), we are clearly in the "non-lattice case" for "almost every" $\alpha$ (in any reasonable sense).

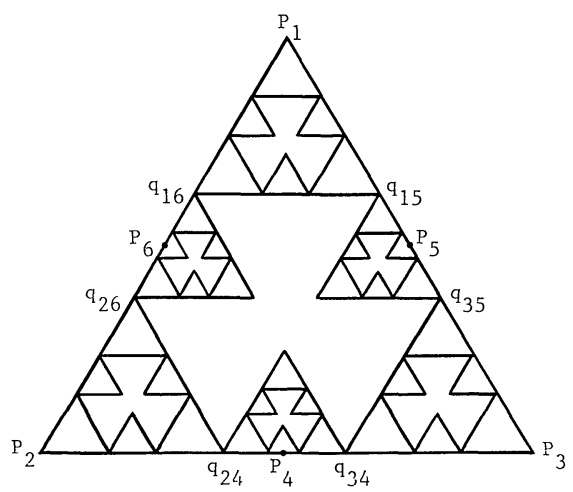

Fig. 2. Modified Sierpinski gasket

\section{Example 4. Hata's Tree-like Set (Fig. 3)}

This tree-like set was introduced by Hata in [Ha].

(I) For a complex number $\beta$ satisfying

$$
|\beta|<1, \quad|1-\beta|<1 \quad \text { and } \quad \operatorname{Im} \beta \neq 0,
$$

we define two contractions on $\mathbb{C}$ by

$$
F_{1}(z)=\beta \bar{z} \quad \text { and } \quad F_{2}(z)=\left(1-|\beta|^{2}\right) \bar{z}+|\beta|^{2} .
$$

The unique compact set $K$ satisfying

$$
K=F_{1}(K) \cup F_{2}(K)
$$

is called Hata's tree-like set. $\left(K,\{1,2\},\left\{F_{\imath}\right\}_{i=1,2}\right)$ is a p.c.f. self-similar structure. In fact,

$$
\begin{gathered}
\mathscr{C}=\{11 \dot{2}, 2 \dot{\mathrm{i}}\}, \quad q=\pi(11 \dot{2})=\pi(2 \dot{\mathrm{i}}) \\
\mathscr{P}=\{\dot{\mathrm{i}}, \dot{2}, 1 \dot{2}\}, \quad p_{1}=\pi(\dot{\mathrm{i}}), \quad p_{2}=\pi(\dot{2}) \quad \text { and } \quad p_{3}=\pi(1 \dot{2}) .
\end{gathered}
$$


(II)

$$
D=\left(\begin{array}{ccc}
-\left(1+\alpha^{-1}\right) & 1 & \alpha^{-1} \\
1 & -1 & 0 \\
\alpha^{-1} & 0 & -\alpha^{-1}
\end{array}\right), \quad r=\left(\alpha, 1-\alpha^{2}\right)
$$

with $0<\alpha<1$. Then $(D, r)$ is a regular harmonic structure where $\lambda=1$.

(III) The spectral exponent $d_{S}$ of the Laplacian associated with $(D, r)$ and a Bernoulli measure $\mu$ is given by

$$
\left(\mu_{1} \alpha\right)^{\frac{d_{S}}{2}}+\left(\mu_{2}\left(1-\alpha^{2}\right)\right)^{\frac{d_{S}}{2}}=1 .
$$

Next, if we choose $\alpha=|\beta|$ and the normalized Hausdorff measure $\mu$ (with $\mu_{1}=\alpha^{d_{H}}$ and $\left.\mu_{2}=\left(1-\alpha^{2}\right)^{d_{H}}\right)$, then (3.5) becomes

$$
\left(\alpha^{\left(d_{H}+1\right)}\right)^{\frac{d_{S}}{2}}+\left(\left(1-\alpha^{2}\right)^{d_{H}+1}\right)^{\frac{d_{S}}{2}}=1 .
$$

Now, since $d_{H}$, the Hausdorff dimension of $K$, is the unique positive number such that $\alpha^{d_{H}}+\left(1-\alpha^{2}\right)^{d_{H}}=1$, we obtain the following remarkable relation between $d_{S}$ and $d_{H}$ :

$$
d_{S}=\frac{2 d_{H}}{d_{H}+1}
$$

Also in this case, the dichotomy between the lattice or non-lattice case, respectively, is that $\log \alpha / \log \left(1-\alpha^{2}\right)$ is rational or not.

Fig. 3. Hata's tree-like set

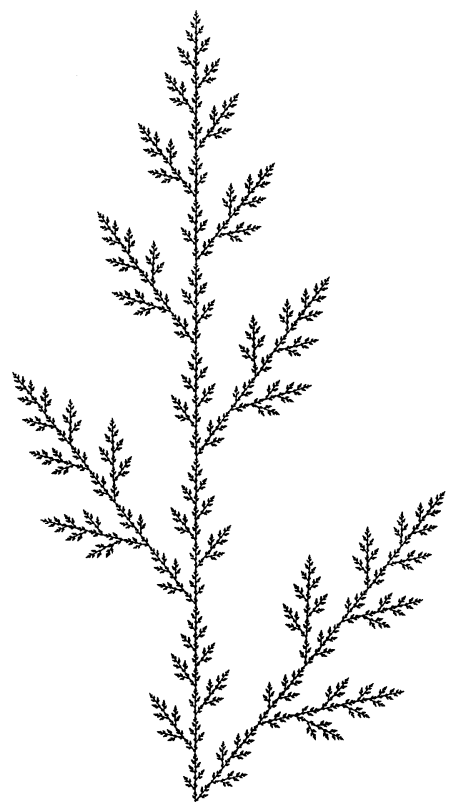

Example 5. Nested Fractals

(I) The "nested fractals" introduced by Lindstrøm in [Li] are p.c.f. self-similar sets $\left(K,\{1,2, \ldots, N\},\left\{F_{\imath}\right\}_{\imath=1,2, \ldots, N}\right)$ such that

$$
K \subset \mathbb{R}^{n} \text { for some } n,
$$


the contractions $F_{i}$ are $\alpha$-similitudes of $\mathbb{R}^{n}$ with the same scaling ratio $\alpha$ (that is, for all $x, y \in \mathbb{R}^{n},\left|F_{\imath}(x)-F_{\imath}(y)\right|=\alpha|x-y|$, where $|\cdot|$ is the Euclidean distance and $0<\alpha<1$ ), and they have strong symmetry.

Further, recall that the Hausdorff dimension $d_{H}$ of $K$ is given by

$$
d_{H}=-\frac{\log N}{\log \alpha}
$$

(II) Using the strong symmetry of $K$, Lindstrøm [Li] has shown that there exists a symmetric decimation invariant random walk on $V_{0}$. In our terminology, this implies that there exists a regular harmonic structure $(D, r)$ with $r=(1,1, \ldots, 1)$ and "renormalization constant" $\lambda$.

(III) If we take, for example, the normalized Hausdorff $\mu$, where $\mu_{i}=\frac{1}{N}$ for $i=1,2, \ldots, N$, then we deduce from (2.2) in Theorem 2.4 that the spectral exponent $d_{S}$ of the associated Laplacian is given by

$$
d_{S}=\frac{2 \log N}{\log (N \lambda)} .
$$

We thus recover a result obtained by Fukushima [Fu2] in the case of the normalized Hausdorff measure. From the probabilistic point of view, the corresponding asymptotic order for the trace of the heat semigroup associated with "Brownian motion" on $K$ was obtained earlier by Lindstrøm in [Li]. Note that in this situation, we are always in the "lattice case" since $\mu_{\imath} r_{\imath} / \lambda=1 / N \lambda$ for all $i=1, \ldots, N$, and thus it follows from Theorem 2.4 that (2.1) and (2.4) hold; in particular, as $x \rightarrow \infty$, we have for the associated Dirichlet Laplacian,

$$
\varrho_{0}(x)=\left(G\left(\frac{\log x}{2}\right)+o(1)\right) x^{\frac{d_{S}}{2}}
$$

where $G$ is the positive periodic function of period $T=1 / \sqrt{N \lambda}$ given by (2.5).

Now, according to Fukushima [Fu2], the integrated density of states for nested fractals is defined by

$$
\mathscr{N}_{0}(x)=\lim _{l \rightarrow \infty} \varrho_{0}\left((N \lambda)^{l} x\right) / N^{l},
$$

where $l \in \mathbb{N}$. It thus follows immediately from Theorem 2.4 that the limit in (3.11) exists and is given by

$$
\mathscr{N}_{0}(x)=G\left(\frac{\log x}{2}\right) x^{\frac{d_{S}}{2}} .
$$

In the above, our results are stated for the Dirichlet Laplacian. According to Corollary 2.5, however, exactly the same results hold for the Neumann Laplacian; in particular, if $\mathscr{N}(x)$ is defined by (3.11) with $\varrho_{0}$ replaced with $\varrho$, the analogue of (3.10) and (3.12) holds for $\varrho(x)$ and $\mathscr{N}(x)$, respectively, where $G$ is the same periodic function as for the Dirichlet problem. In particular, $\mathscr{N}(x)=\mathscr{N}_{0}(x)=G\left(\frac{1}{2} \log x\right) x^{\frac{d_{S}}{2}}$. 


\section{Dirichlet Forms and Dirichlet-Neumann Bracketing}

In this section, we introduce the method of Dirichlet-Neumann bracketing according to Métivier [Me] and Lapidus [La1, Sect. 4,1 pp. 490-497]. It originally treats more general situations of spectrum of certain self-adjoint operators on Hilberts spaces. In this paper, however, we confine our interest to Dirichlet forms. First we recall the definition of Dirichlet forms. (For the theory of Dirichlet forms, see, e.g., Fukushima [Fu1].) Let $X$ be a locally compact separable metric space and let $\nu$ be a regular Borel measure on $X$ such that $\nu(O)>0$ for all open sets $O \subset X$.

Definition 4.1. Let $F$ be a dense subspace of $L^{2}(X, \nu)$ and let $E$ be a non-negative symmetric bilinear form on $F$. Then $(E, F)$ is called a Dirichlet form on $L^{2}(X, \nu)$ if (1) For $\alpha>0$, let $E_{\alpha}(u, v)=E(u, v)+\alpha(u, v)_{\nu}$, where $(u, v)_{\nu}=\int_{X} u v d \nu$. Then,
$\left(F, E_{\alpha}\right)$ is a Hilbert space.

(2) Markov Property: For every $u \in F$, let

$$
\bar{u}(x)= \begin{cases}1 & \text { if } u(x)>1 \\ 0 & \text { if } u(x)<0 \\ u(x) & \text { otherwise }\end{cases}
$$

Then, $\bar{u} \in F$ and $E(\bar{u}, \bar{u}) \leq E(u, u)$.

Remark 1 . It is easy to see that if $\left(F, E_{1}\right)$ is a Hilbert space, then so is $\left(F, E_{\alpha}\right)$ for each $\alpha>0$.

Remark 2. If $(E, F)$ is a Dirichlet form on $L^{2}(X, \nu)$, then $\left(F, L^{2}(X, \nu), E_{\alpha}\right)$ is a variational triple. See, e.g., [La1, Sect. 4.1.A, p. 491] for the definition of a variational triple.

Next we formulate the eigenvalue problem associated with a Dirichlet form.

Definiton 4.2. Let $(E, F)$ be a Dirichlet form on $L^{2}(X, \nu)$. If

$$
E(u, v)=k(u, v)_{\nu}
$$

for all $v \in F$, then $k$ is called an eigenvalue of $(E, F)$ and $u$ is called an eigenfunction belonging to $k$.

Throughout this section, $(E, F)$ denotes a Dirichlet form on $L^{2}(X, \nu)$. Further we assume that the natural inclusion map from the Hilbert space $\left(F, E_{\alpha}\right)$ into $L^{2}(X, \nu)$ is a compact operator. Then it follows from some well-known facts about self-adjoint operators that the eigenvalues of $(E, F)$ are non-negative and of finite multiplicity and that the only accumulation point is $+\infty$.

In this case, the eigenvalues are characterized by the Max-Min principle.

Proposition 4.3. Let $\left\{k_{i}\right\}_{i=1}^{+\infty}$ be the sequence of eigenvalues of $(E, F)$, written according to multiplicity and so that $0 \leq k_{n} \leq k_{n+1}$ for $n=1,2, \ldots$ Then

$$
\left(k_{\imath}+\alpha\right)^{-1 / 2}=d_{i-1}\left(S_{\alpha}(F)\right),
$$

where, for $B \subset L^{2}(X, \nu), S_{\alpha}(B)=\left\{u \in B \cap F \mid E_{\alpha}(u, u) \leq 1\right\}$ and $d_{i}=d_{\imath}(B)$ is defined by

$$
d_{i}=\inf \left\{\sup _{x \in B} \inf _{y \in Y}\|x-y\|_{L^{2}(X, \nu)} \mid Y \text { is an i-dimensional subspace of } L^{2}(X, \nu)\right\} .
$$


Remark. Obviously, if $B \subset Y$ where $Y$ is an $n$-dimensional subspace of $L^{2}(X, \nu)$, then $d_{i}(B)=0$ for $i \geq n$.

The above result yields immediatley an expression for the eigenvalue counting function.

Proposition 4.4. Let us define the eigenvalue counting function $\varrho(x ; E, F)$ by $\varrho(x ; E, F)=\#\left\{i \mid k_{i} \leq x\right\}$. Then

$$
\varrho(x ; E, F)=\#\left\{i \geq 0 \mid d_{i}\left(S_{\alpha}(F)\right) \geq(x+\alpha)^{-1 / 2}\right\} .
$$

Now we consider another Dirichlet form $\left(E^{\prime}, F^{\prime}\right)$ on $L^{2}(X, \nu)$ such that $F^{\prime}$ is a closed subspace of $F$ and $E^{\prime}=\left.E\right|_{F^{\prime} \times F^{\prime}}$. The method of Dirichlet-Neumann bracketing gives a relation between $\varrho(x ; E, F)$ and $\varrho\left(x ; E^{\prime}, F^{\prime}\right)$. By Proposition 4.4, it is easy to see that

\section{Theorem 4.5.}

$$
\varrho\left(x ; E^{\prime}, F^{\prime}\right) \leq \varrho(x ; E, F) .
$$

To estimate the difference between $\varrho(x ; E, F)$ and $\varrho\left(x ; E^{\prime}, F^{\prime}\right)$, we shall need the following refinement of the method of Dirichlet-Neumann bracketing:

Theorem 4.6. [Me]

$$
\varrho(x ; E, F)-\varrho\left(x ; E^{\prime}, F^{\prime}\right)=N\left(x ; E, F, F^{\prime}\right)-\operatorname{dim}\left(F^{\prime} \cap Z_{x}\right),
$$

where $Z_{x}=\left\{u \mid u \in F, E(u, v)=x(u, v)_{\nu}\right.$ for all $\left.v \in F^{\prime}\right\}$ and

$$
N\left(x ; E, F, F^{\prime}\right)=\#\left\{i \mid d_{i-1}\left(S_{\alpha}\left(Z_{x}\right)\right) \geq(x+\alpha)^{-1 / 2}\right\} .
$$

Remark 1. Theorem 4.6 is established in [Me, Proposition 2.7, p. 138]. (See also [La1, Proposition 4.3, p. 493].)

Remark 2. The above refinement of the method of Dirichlet-Neumann bracketing provided by Theorem 4.6 was needed in [La1] to obtain sharp remainder estimates for the spectral asymptotics of elliptic operators on open sets with fractal boundary and will also be necessary in the present paper. (For the original method - which makes use of a special case of inequality (4.1) rather than of the more precise equation (4.2) - we refer to Courant and Hilbert [CH] or Reed and Simon [RS].)

We will use the following special case of Theorem 4.6 in the next section.

Corollary 4.7. If $\operatorname{dim} F / F^{\prime}<+\infty$, then, for all $x$,

$$
\varrho\left(x ; E^{\prime}, F^{\prime}\right) \leq \varrho(x ; E, F) \leq \varrho\left(x ; E^{\prime}, F^{\prime}\right)+\operatorname{dim} F / F^{\prime} .
$$

Remark. The reason why we shall be able to apply Corollary 4.7 is that on a p.c.f. self-similar set, the space of harmonic functions is finite dimensional.

Proof. First, assume that $F^{\prime} \cap Z_{x}=\{0\}$. Let $G$ be the orthogonal complement of $F^{\prime}$ in $F$ with respect to the inner product $E_{1}(\cdot, \cdot)$ and let $p: F \rightarrow G$ be the natural projection. As $F^{\prime} \cap Z_{x}=\{0\}$, it follows that $\left.\operatorname{ker} p\right|_{Z_{x}}=\{0\}$. Hence $\operatorname{dim} Z_{x} \leq \operatorname{dim} G=\operatorname{dim} F / F^{\prime}$. And so by the remark following Proposition 4.3, $N\left(x ; E, F, F^{\prime}\right) \leq \operatorname{dim} F / F^{\prime}$. Using Theorem 4.6 , we thus have

$$
\varrho(x ; E, F) \leq \varrho\left(x ; E^{\prime}, F^{\prime}\right)+\operatorname{dim} F / F^{\prime} .
$$

Now, note that $\left\{x \mid F^{\prime} \cap Z_{x} \neq\{0\}\right\}$ is a countable set because it coincides with the set of eigenvalues of the Dirichlet form $\left(E^{\prime}, F^{\prime}\right)$. Also $\varrho(x ; E, F)$ and $\varrho\left(x ; E^{\prime}, F^{\prime}\right)$ are right continuous. Therefore the above inequality holds for all $x$. 


\section{Laplacians and Dirichlet Forms; the Proof of Lemma 2.3-(2)}

In this section, we will relate eigenvalue problems for Laplacians to eigenvalue problems for Dirichlet forms and prove Lemma 2.3-(2). Recall that $\left(K, S,\left\{F_{\imath}\right\}_{i=1}^{N}\right)$ is a self-similar structure and $(\mathscr{E}, \mathscr{F})$ is a Dirichlet form on $L^{2}(K, \mu)$ constructed by means of a regular harmonic structure $(D, r)$. Also $\Delta_{\mu}$ is the Laplacian associated with $(D, r)$ and $\mu$. The domain of $\Delta_{\mu}$ is denoted by $\mathscr{D}_{\mu}$.

Now, the eigenvalue problem for the Dirichlet form $(\mathscr{E}, \mathscr{F})$ on $L^{2}(K, \mu)$ turns out to be equivalent to the eigenvalue problem for the Laplacian $\Delta_{\mu}$ with homogeneous Neumann boundary conditions as follows.

Proposition 5.1. For $k \in \mathbb{R}$ and $u \in \mathscr{F}$,

$$
\mathscr{E}(u, v)=k(u, v)_{L^{2}(K, \mu)}
$$

for all $v \in \mathscr{F}$ if and only if $u \in \mathscr{D}_{\mu}$ and

$$
\left\{\begin{array}{l}
\Delta_{\mu} u=-k u, \\
(d u)_{p}=0 \text { for all } p \in V_{0} .
\end{array}\right.
$$

Proof. First, assume that for all $v \in \mathscr{F}$,

$$
\mathscr{E}(u, v)=k(u, v)_{L^{2}(K, \mu)} .
$$

Then letting $v=g^{x}$, we have by Proposition 1.9,

$$
u(x)=\sum_{p \in V_{0}} u(p) \psi_{p}(x)+k \int_{K} g(x, y) u(y) \mu(d y) .
$$

Using Theorem 1.12, the above equation implies that $u \in \mathscr{D}$ and $\Delta u=-k u$. Therefore, by Theorem 1.13, we can see that

$$
\mathscr{E}(u, v)=\sum_{p \in V_{0}} v(p)(d u)_{p}+k(u, v)_{L^{2}(K, \mu)}
$$

for all $v \in \mathscr{F}$. Hence we have $(d u)_{p}=0$ for all $p \in V_{0}$.

The converse follows immediately from Theorem 1.13.

For defining the homogeneous Dirichlet boundary conditions, we introduce a new Dirichlet form $\left(\mathscr{E}_{0}, \mathscr{F}_{0}\right)$ as follows.

Proposition 5.2. Let $\mathscr{F}_{0}=\left\{u \in \mathscr{F}|u|_{V_{0}}=0\right\}$ and let $\mathscr{E}_{0}=\mathscr{E}_{\mathscr{F}_{0} \times \mathscr{F}_{0}}$. Then $\left(\mathscr{E}_{0}, \mathscr{F}_{0}\right)$ is a local regular Dirichlet form on $L^{2}(K, \mu)$. Further, for $k \in \mathbb{R}$ and $u \in \mathscr{F}_{0}$,

$$
\mathscr{E}_{0}(u, v)=k(u, v)_{L^{2}(K, \mu)}
$$

for all $v \in \mathscr{F}_{0}$ if and only if $u \in \mathscr{D}_{0}=\mathscr{D}_{\mu} \cap \mathscr{F}_{0}$ and

$$
\left\{\begin{array}{l}
\Delta_{\mu} u=-k u \\
\left.u\right|_{V_{0}}=0 .
\end{array}\right.
$$

Proof. Note that $\mathscr{F}=\mathscr{F}_{0}+H$, where $H$ is the set of harmonic functions, and that $\operatorname{dim} H=\#\left(V_{0}\right)$. Hence it is easy to check that $\mathscr{F}_{0}$ is dense in $L^{2}(K, \mu)$. Further, the other conditons follow immediately from the definition of $\left(\mathscr{E}_{0}, \mathscr{F}_{0}\right)$. And so $\left(\mathscr{E}_{0}, \mathscr{F}_{0}\right)$ is 
a local regular Dirichlet form on $L^{2}(K, \mu)$. The proof of the second part of the above proposition is similar to that of Proposition 5.1.

Now we proceed to give the proof of Lemma 2.3-(2). By the above propositions,

$$
\varrho(x)=\varrho(x ; \mathscr{E}, \mathscr{F}) \text { and } \varrho_{0}(x)=\varrho\left(x ; \mathscr{E}_{0}, \mathscr{F}_{0}\right),
$$

where we use the notation of Proposition 4.4. And thus we can apply the method of Dirichlet-Neumann bracketing if the natural inclusion map $\mathscr{F} \hookrightarrow L^{2}(K, \mu)$ is shown to be a compact operator.

Lemma 5.3. There exists a positive constant $C$ such that, for all $u \in \mathscr{F}$,

$$
C\|u\|_{\infty}^{2} \leq \mathscr{E}(u, u)+\|u\|_{2}^{2},
$$

where $\|u\|_{\infty}=\sup _{x \in K}|u(x)|$ and $\|u\|_{2}=\sqrt{\int_{K} u^{2} d \mu}$.

Proof. By Proposition 1.9, we have

$$
\sqrt{\mathscr{E}\left(g^{x}, g^{x}\right)} \sqrt{\mathscr{E}(u, u)} \geq\left|\mathscr{E}\left(g^{x}, u\right)\right|=|u(x)-\bar{u}(x)|,
$$

where $\bar{u}(x)=\sum_{p \in V_{0}} u(p) \psi_{p}(x)$. Also by Propositon 1.9, $\mathscr{E}\left(g^{x}, g^{x}\right)=g(x, x)$. Hence
we have

$$
\|u-\bar{u}\|_{\infty}^{2} \leq C_{1} \mathscr{E}(u, u)
$$

where $C_{1}=\sup _{x \in K} g(x, x)$.

Now note that $\bar{u} \in H$. As $H$ is finite dimensional, the norms $\|\cdot\|_{\infty}$ and $\|\cdot\|_{2}$ are mutually equivalent. Therefore, there exists a positive constant $C_{2}$ such that, for all $u \in \mathscr{F}$,

$$
\|\bar{u}\|_{\infty} \leq C_{2}\|\bar{u}\|_{2} \leq C_{2}\left(\|u\|_{2}+\|u-\bar{u}\|_{2}\right)
$$

Since $\|\cdot\|_{\infty} \geq\|\cdot\|_{2}$, we obtain, for some $C_{3}>0$,

$$
\|\bar{u}\|_{\infty} \leq C_{3}\left(\|u\|_{2}+\sqrt{\mathscr{E}(u, u)}\right) .
$$

Since $\|\bar{u}\|_{\infty}+\|u-\bar{u}\|_{\infty} \geq\|u\|_{\infty}$, the proof of Lemma 5.3 is complete.

Lemma 5.4. The natural inclusion map $\mathscr{F} \hookrightarrow L^{2}(K, \mu)$ is a compact operator.

Proof. Let $U$ be a bounded set in $\mathscr{F}$ with respect to the inner product $\mathscr{E}(u, v)+$ $\int_{K} u v d \mu$. Then by Lemma 5.3, there exists a positive constant $A$ such that, for all $u \in U,\|u\|_{\infty} \leq A$. Hence $U$ is uniformly bounded.

On the other hand, using Proposition 1.9, we have

$$
\mathscr{E}\left(g^{x}-g^{y}, u\right)=u(x)-u(y)+\left(\sum_{p \in V_{0}} u(p)\left(\psi_{p}(x)-\psi_{p}(y)\right)\right) .
$$

Hence we deduce that

$$
\sqrt{\mathscr{E}\left(g^{x}-g^{y}, g^{x}-g^{y}\right) \mathscr{E}(u, u)}+A \#\left(V_{0}\right) \max _{p \in V_{0}}\left|\psi_{p}(x)-\psi_{p}(y)\right| \geq|u(x)-u(y)| .
$$

Since $\mathscr{E}\left(g^{x}-g^{y}, g^{x}-g^{y}\right)=g(x, x)+g(y, y)-2 g(x, y)$ and $\mathscr{E}(u, u)$ are bounded, we conclude that $U$ is equicontinuous.

Thus it follows from the Ascoli-Arzelà theorem that $U$ is relatively compact in $C(K)$. And hence $U$ is relatively compact in $L^{2}(K, \mu)$. 
Proof. Lemma 6.1 and the definitions imply (1) and (2) immediately.

(3) Let $U \subset \tilde{\mathscr{F}}$ be bounded in $(\tilde{\mathscr{E}}, \tilde{\mathscr{F}})$. Then, for each $i=1,2, \ldots, N, U_{i}=$ $\left\{f \circ F_{\imath} \mid f \in U\right\}$ is a bounded set in $(\mathscr{E}, \mathscr{F})$. And so it is relatively compact in $L^{2}(K, \mu)$. Hence $U$ is relatively compact in $L^{2}(K, \mu)$.

(4) Let $f$ be an eigenfunction of the Dirichlet form $(\tilde{\mathscr{E}}, \tilde{\mathscr{F}})$ with eigenvalue $k$. Then by the definitions, for all $g \in \tilde{\mathscr{F}}$,

$$
\lambda \sum_{i=1}^{N} r_{i}^{-1} \mathscr{E}\left(f_{\imath}, g_{i}\right)=\tilde{\mathscr{E}}(f, g)=k(f, g)_{L^{2}(K, \mu)}=k \sum_{i=1}^{N} \mu_{i} \int_{K} f_{i} g_{i} d \mu .
$$

Hence we have for any $h \in \mathscr{F}$,

$$
\frac{\lambda}{r_{i}} \mathscr{E}\left(f_{i}, h\right)=k \mu_{i}\left(f_{i}, h\right)_{L^{2}(K, \mu)} .
$$

Therefore, for each $i, g_{i}=f \circ F_{\imath}$ is an eigenfunction of the Dirichlet form $(\mathscr{E}, \mathscr{F}$ ) with eigenvalue $\frac{\mu_{i} r_{\imath}}{\lambda} k$. The converse is obvious and hence we have

$$
\varrho(x ; \tilde{\mathscr{E}}, \tilde{\mathscr{F}})=\sum_{i=1}^{N} \varrho\left(\frac{\mu_{i} r_{i}}{\lambda} x\right) .
$$

Using Theorem 4.5, the above proposition implies a part of Lemma 2.3-(1), that is

$$
\varrho(x) \leq \sum_{i=1}^{N} \varrho\left(\frac{\mu_{\imath} r_{\imath}}{\lambda} x\right) .
$$

To obtain the remaining part, we introduce another Dirichlet form on $L^{2}(K, \mu)$.

Proposition 6.3. Let $\tilde{\mathscr{F}}=\left\{f\left|f \in \mathscr{F}_{0}, f\right|_{V_{1}}=0\right\}$ and let $\tilde{\mathscr{E}}_{0}=\left.\mathscr{E}\right|_{\tilde{\tilde{K}} \times \tilde{\tilde{\pi}}}$. Then

(1) $\left(\tilde{\mathscr{E}}_{0}, \tilde{\mathscr{F}_{0}}\right)$ is a local regular Dirichlet form on $L^{2}(K, \mu)$.

(2) $\varrho\left(x ; \tilde{\mathscr{E}}_{0}, \tilde{\mathscr{F}}\right)=\sum_{i=1}^{N} \varrho_{0}\left(\frac{\mu_{i} r_{i}}{\lambda} x\right)$.

The proof of this proposition is entirely similar to that of Proposition 6.2.

Finally, we deduce from Theorem 4.5 that

$$
\sum_{i=1}^{N} \varrho_{0}\left(\frac{\mu_{i} r_{i}}{\lambda} x\right) \leq \varrho_{0}(x)
$$

Hence we have completed the proof of Lemma 2.3-(1).

\section{Appendix: The Spectral Dimension}

In Sect. 2, we have established a formula to calculate the spectral exponent $d_{S}$ of a Laplacian $\Delta_{\mu}$ associated with a self-similar structure $(K,\{1,2, \ldots, N\}$, $\left.\left\{F_{\imath}\right\}_{i=1,2, \ldots, N}\right)$, a regular harmonic structure $(D, r)$ and a Bernoulli measure $\mu$ on $K$. The formula is

$$
\sum_{i=1}^{N} \gamma_{i}^{d_{S}}=1
$$


Remark. It follows from the above proof that the inclusion map $\mathscr{F} \hookrightarrow C(K)$ is a compact operator.

Finally, using Corollary 4.7, we have

$$
\varrho_{0}(x) \leq \varrho(x) \leq \varrho_{0}(x)+\#\left(V_{0}\right),
$$

because $\#\left(V_{0}\right)$ is finite and $\operatorname{dim} \mathscr{F} / \mathscr{F}_{0}=\operatorname{dim} H=\#\left(V_{0}\right)$. Hence we obtain Lemma 2.3-(2).

\section{Scaling Property of Dirichlet Forms; the Proof of Lemma 2.3-(1)}

In this section, we will prove Lemma 2.3(1). The key is a kind of scaling property of the Dirichlet form $(\mathscr{E}, \mathscr{F})$, as given below.

Lemma 6.1. For $u, v \in \mathscr{F}$,

$$
\mathscr{E}(u, v)=\lambda \sum_{i=1}^{N} r_{i}^{-1} \mathscr{E}\left(u \circ F_{i}, v \circ F_{i}\right) .
$$

Proof. By Definition 1.5 (and with the obvious simplified notation),

$$
\mathscr{E}_{m}\left(u \circ F_{\imath}, v \circ F_{\imath}\right)=-\lambda^{m} \sum_{w \in W_{m}} r_{w}^{-1 t}\left(R_{w}\left(u \circ F_{i}\right)\right) D\left(R_{w}\left(v \circ F_{\imath}\right)\right) .
$$

Note that $R_{w}\left(f \circ F_{i}\right)=R_{i w} f$; hence the above equality implies

$$
\begin{aligned}
\lambda \sum_{\imath=1}^{N} r_{\imath}^{-1} \mathscr{E}_{m}\left(u \circ F_{i}, v \circ F_{i}\right) & =-\lambda^{m+1} \sum_{\imath=1}^{N} \sum_{w \in W_{m}} r_{i w}^{-1}\left(R_{\imath w} u\right) D\left(R_{\imath w} v\right) \\
& =\mathscr{E}_{m+1}(u, v) .
\end{aligned}
$$

In view of Proposition 1.7, we complete the proof by letting $m \rightarrow \infty$.

Remark. This kind of scaling property of Dirichlet forms on self-similar sets was first established by Fukushima [Fu2] for nested fractals.

Now we introduce a new Dirichlet form on $L^{2}(K, \mu)$.

Proposition 6.2. Let $\tilde{\mathscr{F}}$ be defined by

$\tilde{\mathscr{F}}=\left\{f: K \backslash V_{1} \rightarrow \mathbb{R} \mid\right.$ for $i=1,2, \ldots, N, f \circ F_{i}=f_{i}$ on $K \backslash V_{0}$ for some $\left.f_{i} \in \mathscr{F}\right\}$.

Let $\tilde{\mathscr{E}}$ be a nonnegative symmetric form on $\tilde{\mathscr{F}}$ defined by

$$
\tilde{\mathscr{E}}(f, g)=\lambda \sum_{i=1}^{N} r_{i}^{-1} \mathscr{E}\left(f_{i}, g_{i}\right)
$$

Then

(1) $\mathscr{F} \subset \tilde{\mathscr{F}}$ and $\mathscr{E}=\left.\tilde{\mathscr{E}}\right|_{\mathscr{F} \times \mathscr{F}}$.

(2) $(\tilde{\mathscr{E}}, \tilde{\mathscr{F}})$ is a local regular Dirichlet form on $L^{2}(K, \mu)$.

(3) The natural inclusion map $\tilde{\mathscr{F}} \hookrightarrow L^{2}(K, \mu)$ is a compact operator.

(4) $\varrho(x ; \tilde{\mathscr{E}}, \tilde{\mathscr{F}})=\sum_{i=1}^{N} \varrho\left(\frac{\mu_{\imath} r_{i}}{\lambda} x\right)$. 
where $\gamma_{i}=\sqrt{\frac{r_{i} \mu_{i}}{\lambda}}$ for $i=1,2, \ldots, N$. In this appendix, we will obtain a relation between this spectral exponent and the so-called spectral dimension. We will fix a selfsimilar structure and a regular harmonic structure and think of the spectral exponent as a function of $\mu$. And so we write $d_{S}=d_{s}(\mu)$. Fist we will define the similarity dimension of the harmonic structure.

Definition A.1. The unique positive number $S$ which satisfies

$$
\sum_{i=1}^{N}\left(\frac{r_{i}}{\lambda}\right)^{S}=1
$$

is called the similarity dimension of the harmonic structure $(D, r)$.

Then we deduce the following theorem from an elementary calculation.

Theorem A.2.

$$
\max \left\{d_{S}(\mu): \mu \text { is a Bernoulli measure on } K\right\}=\frac{2 S}{S+1},
$$

where the maximum is attained only at the Bernoulli measure $\mu$ such that

$$
\mu_{i}=\left(\frac{r_{i}}{\lambda}\right)^{S}, \text { for } i=1, \ldots, N .
$$

The positive number

$$
d_{S}^{*}=\frac{2 S}{S+1}
$$

defined by (A.3), with $S$ given by (A.2), is called the spectral dimension of the harmonic structure $(D, r)$.

Proof. We can determine the maximum of $d_{S}(\mu)$ as a function of $\left(\mu_{1}, \ldots, \mu_{N}\right)$, subject to the constraints $\sum_{i=1}^{N} \mu_{i}=1, \mu_{\imath} \geq 0(i=1, \ldots, N)$, by using the method of Lagrange multipliers.

Remark 1. Clearly, we always have $d_{S}^{*}<2$. Further, $d_{S}^{*}=S$ if and only if $S=1$. (This is of interest in view of Remarks 2 and 3 following Corollary 2.5.)

Remark 2. Fujita [Fj1,2] has studied generalized diffusions on an interval associated with self-similiar measures and has obtained the corresponding relation between the similarity dimension and the spectral dimension as above. This result indicates that our relation could be extended to generalized diffusions on fractals.

Remark 3. Our results suggest certain formal analogies with the interesting work of Strichartz [St1,2] on the "Fourier asymptotics of (self-similar) fractal measures" in $\mathbb{R}^{n}$.

Now we revisit some of the examples discussed in Sect. 3. (For simplicity, we number the examples in the same way as in Sect. 3.)

Example 1. (Interval.) For the harmonic structure $(D, r)$ where

$$
D=\left(\begin{array}{cc}
-1 & 1 \\
1 & -1
\end{array}\right) \text { and } r=(\alpha, 1-\alpha) \text {, }
$$

the similarity dimension is 1 . Hence the spectral dimension of $(D, r)$ is 1 . 
Example 2. (Sierpinski Gasket.) For the harmonic structure $(D, r), \frac{r_{i}}{\lambda}=\frac{3}{5}$. Hence the similarity dimension is $\log 3 /(\log 5-\log 3)$ and the spectral dimension is $\log 9 / \log 5$. This value is the so-called "spectral dimension of the Sierpinski gasket."

More generally, for the $N$-Sierpinski gasket studied in Example 2 of Sect. 3, we have $\frac{r_{i}}{\lambda}=\frac{N}{N+2}$, and hence

$$
S=\frac{\log N}{\log (N+2)-\log N} \quad \text { and } \quad d_{S}^{*}=\frac{2 \log N}{\log (N+2)} .
$$

Example 4. (Hata's Tree-like Set.) For the harmonic structure $(D, r)$, where

$$
D=\left(\begin{array}{ccc}
-\left(1+\alpha^{-1}\right) & 1 & \alpha^{-1} \\
1 & -1 & 0 \\
\alpha^{-1} & 0 & \alpha^{-1}
\end{array}\right) \quad \text { and } r=\left(\alpha, 1-\alpha^{2}\right)
$$

the similarity dimension is given by

$$
\alpha^{S}+\left(1-\alpha^{2}\right)^{S}=1 \text {. }
$$

If we choose $\alpha=|\beta|$, then $S$ equals the Hausdorff dimension $d_{H}$, where the contractions are

$$
F_{1}(z)=\beta \bar{z} \quad \text { and } \quad F_{2}(z)=\left(1-|\beta|^{2}\right) \bar{z}+|\beta|^{2} .
$$

Further, the Bernoulli measure which gives the spectral dimension of $(D, r)$ is the natural (normalized) Hausdorff measure with respect to the above contractions. In this case, we have already stated the relation between the similarity dimension and the spectral dimension in Sect. 3; namely, (A.5) yields

$$
d_{S}^{*}=\frac{2 d_{H}}{d_{H}+1}
$$

as was obtained in (3.7).

Example 5. (The Nested Fractals.) For the harmonic structure $(D,(1,1, \ldots, 1))$, the similarity dimension is given by

$$
S=\frac{\log N}{\log \lambda}
$$

Hence, by (A.5), the spectral dimension $d_{S}^{*}$ is given by

$$
d_{S}^{*}=\frac{2 \log N}{\log (N \lambda)}
$$

where the corresponding measure $\mu$ is characterized by $\mu_{i}=\frac{1}{N}$, for $i=1, \ldots, N$. In particular, we recover formula (3.9) obtained in Example 5 of Sect. 3. Note that in this case, $\mu$ is the natural (normalized) Hausdorff measure on $K$.

Acknowledgements. Michel L. Lapidus woul like to thank Professor Tim Bedford for providing him with a helpful reference and Professor Robert S. Strichartz for a comment on the manuscript. He is also grateful to the Department of Mathematics of Yale University for its hospitality while he was a Visiting Professor during the preliminary stages of this work. he would especially like to express his appreciation to his sponsors at Yale, Professor Raphael C. Coifman and Professor Benoit B. Mandelbrot, for their kind welcome in New Haven. 


\section{References}

[AO] Alexander, S., Orbach, R.: Density of states on fractals: fractons. J. Physique Lettres 43, L625-L631 (1982)

[Ba] Barlow, M.T.: Random walks and diffusion on fractals. In: Proc. Intern. Congress Math. (Kyoto, 1990), vol. II, Berlin, Heidelberg, New York: Springer 1991, pp. 1025-1035

[BB1] Barlow, M.T., Bass, R.F.: The construction of Brownian motion on the Sierpinski carpet. Ann. Inst. Henri Poincaré 25, 225-257 (1989)

[BB2] Barlow, M.T., Bass, R.F.: Local time for Brownian motion on the Sierpinski carpet. Probab. Theory Related Fields 85, 91-104 (1990)

[BB3] Barlow, M.T., Bass, R.F.: On the resistance of the Sierpinski carpet. Proc. R. Soc. Lond. Ser. A 431, 354-360 (1990)

[BB4] Barlow, M.T., Bass, R.F.: Transition densities for Brownian motion on the Sierpinski carpet. Preprint 1991

[BBS] Barlow, M.T., Bass, R.F., Sherwood, J.D.: Resistance and spectral dimension of Sierpinski carpets. J. Physique Lettres A 23, L253-L258 (1990)

[BP] Barlow, M.T., Perkins, E.A.: Brownian motion on the Sierpinski gasket. Probab. Theory Related Fields 79, 542-624 (1988)

[Be1] Berry, M.V.: Distribution of modes in fractal resonators. In: Structural stability in physics. Güttinger, W., Eikemeier, H. (eds.), Berlin, Heidelberg, New York: Springer 1979, pp. 51-53

[Be2] Berry, M.V.: Some geometric aspects of wave motion: wavefront dislocations, diffraction catastrophes, diffractals. In: Geometry of the Laplace operator, Proc. Symp. Pure Math., vol. 36, Providence, R.I.: Am. Math. Soc. 1980, pp. 13-38

[BC] Brossard, J., Carmona, R.: Can one hear the dimension of a fractal? Commun. Math. Phys. 104, 103-122 (1986)

[CH] Courant, R., Hilbert, D.: Methods of mathematical physics. vol. I, English transl., New York: Interscience 1953

[D] Dhar, D.: Lattices of effectively nonintegral dimensionality. J. Math. Phys. 18, 577-585 (1977)

[Fa] Falconer, K.J.: Fractal geometry: mathematical foundations and applications. Chichester: Wiley 1990

[Fe] Feller, W.: An introduction to probability theory and its applications. Vol. II. New York: Wiley 1966

[Fj1] Fujita, T.: A fractional dimension, self-similarity and a generalized diffusion operator. In: Probabilistic methods in mathematical physics, Proc. Taniguchi Intern. Symp. (Katata and Kyoto, 1985), Ito, K., Ikeda, N. (eds.), Toyko: Kinokuniya 1987, pp. 83-90

[Fj2] Fujita, T.: Some asymptotics estimates of transition probability densities for generalized diffusion processes with self-similar speed measures. Preprint

[Fu1] Fukushima, M.: Dirichlet forms and Markov processes. Amsterdam: North-Holland/Kodansya 1980

[Fu2] Fukushima, M.: Dirichlet forms, diffusion processes and spectral dimensions for nested fractals. In: Ideas and methods in mathematical analysis, stochastics and applications, Proc. Conf. in Memory of Hoegh-Krøhn, Vol. 1, Albeverio, S., et al. (eds.), Cambridge: Cambridge Univ. Press 1992 (to appear)

[FS] Fukushima, M., Shima, T.: On a spectral analysis for the Sierpinski gasket. Potential Analysis 1, 1-35 (1992)

[G] Goldstein, S.: Random walks and diffusions on fractals. In: Percolation theory and ergodic theory of infinite particle systems. IMA Math. Appl., Vol. 8, Kesten, H. (ed.), Berlin, Heidelberg, New York: Springer 1987, pp. 121-129

[Ha] Hata, M.: On the structure of self-similar sets. Jpn. J. Appl. Math. 3, 381-414 (1985)

[HHW] Hattori, K., Hattori, T., Watanabe, H.: Gaussian field theories on general networks and the spectral dimensions. Progr. Theoret. Phys. Suppl. 92, 108-143 (1987)

[HB] Havlin, S., Bunde, A.: Percolation II (Chap. 3). In: Fractals and disordered systems, Berlin, Heidelberg, New York: Springer 1991, pp. 97-149

[Hu] Hutchinson, J.E.: Fractals and self-similarity. Indiana Univ. Math. J. 30, 713-843 (1981) 
[Ka] Kameyama, A.: Self-similar sets from the topological point of view. Jpn. J. Indust. Appl. (to appear)

[Ki1] Kigami, J.: A harmonic calculus on the Sierpinski spaces. Jpn. J. Appl. Math. 6, 259-290 (1989)

[Ki2] Kigami, J.: Harmonic calculus on p.c.f. self-similar sets. Trans. Am. Math. Soc. 335, 721755 (1993)

[Ki3] Kigami, J.: Harmonic metric and Dirichlet form on the Sierpinski gasket. In: Asymptotic problems in probability theory, Elworthy, K.D., Ikeda, N. (eds.). Pitman (to appear)

[Km1] Kumagai, T.: Analysis of diffusion processes on finitely ramified fractals. Master Thesis, Kyoto Univ., Kyoto, Japan 1991

[Km2] Kumagai, T.: Regularity, closedness and spectral dimensions of the Dirichlet forms on p.c.f. self-similar sets. J. Math. Kyoto Univ. (to appear)

[Ku1] Kusuoka, S.: A diffusion process on a fractal. In: Probabilistic methods in mathematical physics, Proc. Taniguchi Intern. Symp. (Katata and Kyoto, 1985), Ito, K., Ikeda, N. (eds.). Tokyo: Kinokuniya 1987, pp. 251-274

[Ku2] Kusuoka, S.: Dirichlet forms on fractals and products of random matrices. Publ. Res. Inst. Math. Sci. 25, 659-680 (1989)

[L] Lalley, L.P.: Packing and covering functions of some self-similar fractals. Indiana Univ. Math. J. 37, 699-709 (1988)

[La1] Lapidus, M.L.: Fractal drum, inverse spectral problems for elliptic operators and a partial resolution of the Weyl-Berry conjecture. Trans. Am. Math. Soc. 325, 465-529 (1991)

[La2] Lapidus, M.L.: Can one hear the shape of a fractal drum? Partial resolution of the WeylBerry conjecture. In: Geometric analysis and computer graphics (MSRI, Berkeley, 1988), Mathematical Sciences Research Institute Publications, Vol. 17, Concus, P., et al. (eds.), Berlin, Heidelberg, New York: Springer 1991, pp. 119-126

[La3] Lapidus, M.L.: Spectral and fractal geometry: From the Weyl-Berry conjecture for the vibrations of fractal drums to the Riemann zeta-function. In: Differential equations and mathematical physics, Proc. UAB Intern. Conf. on differential equations and mathematical physics (Birmingham, 1990), Bennewitz, C. (ed.), New York: Academic Press 1992, pp. 151182

[La4] Lapidus, M.L.: Vibrations of fractal drums, the Riemann hypothesis, waves in fractal media, and the Weyl-Berry conjecture. In: Ordinary and partial differential equations, Vol. IV, Proc. Twelvth Intern. Conf. on the theory of ordinary and partial differential equations (Dundee, Scotland 1992) Research Notes in Mathematics Series, Sleeman, B.D., Jarvis, R.J. (eds.). London: Longman Group UK Limited 1993, pp. 126-209

[La5] Lapidus, M.L.: Can one hear the shape of a fractal drum? From the Weyl-Berry conjecture to the Riemann hypothesis. Mathematical Intelligencer (to appear). [Expanded version of a plenary address given at the Regional Meeting of the American Mathematical Society in Tampa, Florida, in March 1991.]

[LF] Lapidus, M.L., Fleckinger-Pellé, J.: Tambour fractal: Vers une résolution de la conjecture de Weyl-Berry pour les valeurs propres du laplacien. C.R. Acad. Sci. Paris Sér. I Math. 306, 171-175 (1988)

[LM1] Lapidus, M.L., Maier, H.: Hypothèse de Rieman, cordes fractals vibrantes et conjecture de Weyl-Berry modifiée. C.R. Acad. Sci. Paris Sér, I Math. 313, 19-24 (1991)

[LM2] Lapidus, M.L., Maier, H.: The Riemann hypothesis and inverse spectral problems for fractal strings. J. Lond. Math. Soc. (to appear)

[LP1] Lapidus, M.L., Pomerance, C.: Fonction zêta de Riemann et conjecture de Weyl-Berry pour les tambours fractals. C.R. Acad. Sci. Paris Sér, I Math. 310, 343-348 (1990)

[LP2] Lapidus, M.L., Pomerance, C.: The Riemann zeta-function and the one-dimensional WeylBerry conjecture for fractal drums. Proc. Lond. Math. Soc. (3) 66, No. 1, 41-69

[LP3] Lapidus, M.L., Pomerance, C.: Spectral zeta-functions and the $n$-dimensional Weyl-Berry problem for fractal drums (to appear)

[Li] Lindstrøm, T.: Brownian motion on nested fractals. Mem. Am. Math. Soc. No. 420, 83 (1990)

[Liu] Liu, S.H.: Fractals and their applications in condensed matter physics. Solid State Phys. 39, 207-273 (1986)

[Me] Métivier, G.: Valeurs propres de problèmes aux limites elliptiques irréguliers. Bull. Soc. Math. France, Mém. 51-52, 125-219 (1977) 
[Mo] Moran, P.A.P.: Additive functions of intervals and Hausdorff measure. Proc. Camb. Phil. Soc. 42, 15-23 (1946)

[Ra] Rammal, R.: Spectrum of harmonic excitations on fractals. J. Physique 45, 191-206 (1984)

[RT] Rammal, R., Toulouse, G.: Random walks on fractal structures and percolation clusters. J. Physique Lettres 44, L13-L22 (1983)

[RS] Reed, M., Simon, B.: Methods of modern mathematical physics. Vol. IV, Analysis of operators. New York: Academic Press 1978

[Ru] Rudin, W.: Functional analysis. New Delhi: Tata McGraw-Hill 1974

[Sc] Schroeder, M.: Fractals, chaos, power laws. New York: Freeman 1991

[Sh] Shima, T.: On eigenvalue problems for the random walks on the Sierpinski pre-gaskets. Jpn. J. Indust. Appl. Math. 8, 124-141 (1991)

[St1] Strichartz, R.S.: Fourier asymptotics of fractal measures. J. Funct. Anal. 89, 154-187 (1990)

[St2] Strichartz, R.S.: Self-similar measures and their Fourier transforms, I-III. I, Indiana Univ. Math. J. 39, 797-817 (1990); II, Trans. Am. Math. Soc. (to appear); III, Preprint 1992

[W1] Weyl, H.: Über die asymptotische Verteilung der Eigenwerte. Gött. Nach., 110-117 (1911)

[W2] Weyl, H.: Das asymptotische Verteilungsgesetz der Eigenwerte linearer partieller Differentialgleichungen. Math. Ann. 71, 441-479 (1912)

Communicated by H. Araki 
